\title{
Essai de prévision des étiages de l'Oise à Sempigny
}

\author{
PAR
}

\section{A. Guilbot}

J.-M. Masson

Laboratoire d'Hydrologie de Montpellier

\author{
G. Bédiot
}

ET C. Ducastelle

Agence Financière de Bassin Seine-Normandie

\section{Introduction}

La prévision des étiages devient une nécessité. Les basses eaux sont en effet à l'origine de deux sortes de risques pour les usagers de l'eau :

a) la pénurie d'eau liée au degré de sévérité de l'étiage défini par sa durée et ses débits les plus faibles,

b) l'élévation des taux de concentration de produits polluants, dépendant à la fois du cours d'eau et de l'activité économique du bassin.

Ces deux risques ne sont pas forcément concomitants.

A titre exploratoire, l'Agence Financière de Bassin Seine-Normandie a demandé à la S.M.L.R.S. une étude destinée à tester l'efficacité de différentes méthodes de prévision dans un cas bien précis : celui de l'Oise à Sempigny. Ce bassin a été choisi parce qu'il est de superficie moyenne (4290 km²), qu'il se situe en amont d'une zone industrialisée (basse vallée de l'Oise et Région Parisienne) ayant d'importants besoins en eau et qu'il n'est pas influencé par des lâchures de barrage-réservoir. Enfin, une étude précédente (Lavabre, Masson et Saurin) a permis de constater que la qualité des données anciennes de débits à Sempigny était acceptable.

La précision ne porte que sur les grandeurs suivantes : débits minimaux de 5 à 10 jours consécutifs d'un mois donné, débit moyen mensuel. Elle est émise deux mois auparavant : par exemple le ler Juin pour le mois d'Août. Aucun pronostic n'est fait sur la durée de l'étiage qui est un terme essentiel de sa sévérité.

\section{L'OISE A SEMPIGNY}

PLAN dE SITUATION DE L'ETUdE

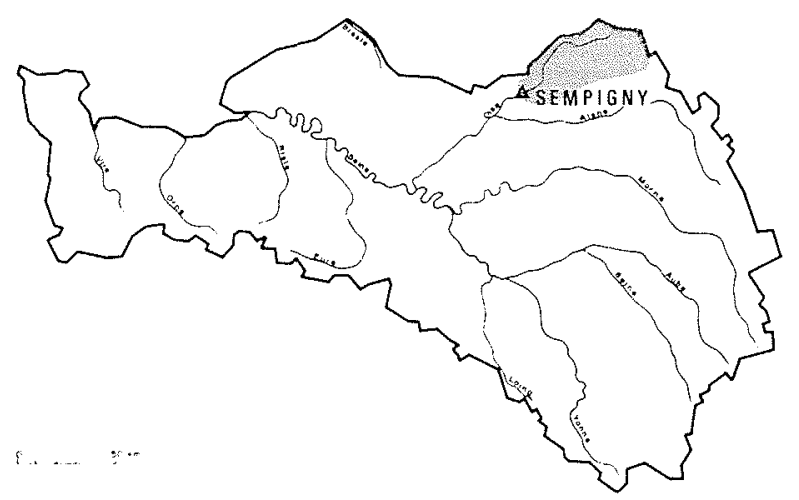

Carte 1/

\section{Les données utilisées}

\subsection{Les débits}

Les débits moyens journaliers de l'Oise à Sempigny (4290 $\mathrm{km}^{2}$ ) sont mesurés et publiés depuis 1955 par la C.E. Nord et Paris.

La station est stable et la courbe de tarage est bien connue de part et d'autre de la cote de débordement.

On peut toutefois remarquer: 


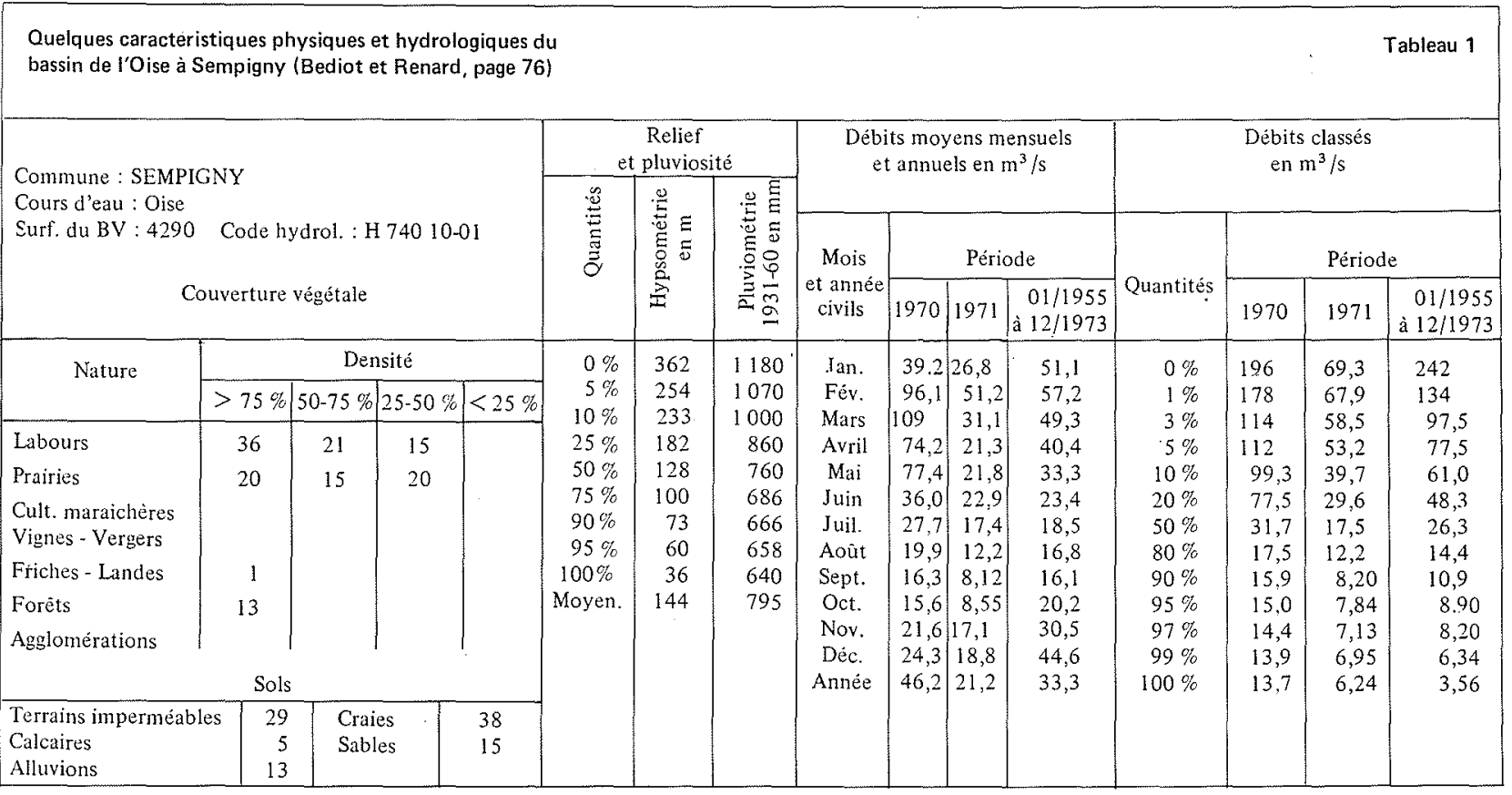

- que les très faibles valeurs de débits, obtenues lors de jaugeages, présentent une assez forte dispersion autour de la courbe théorique ;

- que les débits à Sempigny ne représentent pas exactement le débit naturel de l'Oise et que deux ouvrages interviennent de façon non négligeable mais approximativement constante,

- le canal latéral à l'Oise dont on peut chiffrer les prélèvements sur le bassin de l'Oise à environ $0,3 \mathrm{~m}^{3} / \mathrm{s}$ auxquels s'ajoutent $0,3 \mathrm{~m}^{3} / \mathrm{s}$ en provenance de la Sambre,

- la rigole de l'Oise et du Noirieu qui opère vers la Somme un transfert moyen de $1 \mathrm{~m}^{3} / \mathrm{s}$.

Les hauteurs d'eau sont observées quotidiennement depuis 1892 à l'échelle du service hydrologique d'annonce des crues (S.H.A.C.), avec une seule interruption pendant la première guerre mondiale. La courbe de tarage de la station de la circonscription électrique a été appliquée aux lectures quotidiennes de l'échelle du S.H.A.C. Les débits ainsi reconstitués ont été jugés à priori représentatifs des débits moyens joumaliers de l'Oise à Sempigny. Une vérification de l'homogénéité de la série reconstituée a été réalisée par double cumul avec les précipitations.

\subsection{Les précipitations}

\subsubsection{Données existantes}

Le réseau pluviométrique ne devient suffisamment dense qu'en 1971 avec la création du réseau pluviométrique complémentaire de Seine-Normandie.

Avant cette date, 15 stations au maximum ont pu être utilisées de façon concomitante pour la période 19571970. Pour les années antérieures, un minimum de 6 stations a pu être utilisé (voir carte 2).

\subsubsection{Critique des données et estimation des lames d'eau précipitées}

Une critique des quinze séries d'informations pluviométriques suffisamment longues a été téalisée : d'une part, par double cumul, d'autre part par cumul des résidus.

Bien que quelques hétérogénéités ponctuelles apparaissent, aucun poste n'a finalement été éliminé.

A partir de ces éléments, nous avons cherché à obtenir mois par mois sur la période d'Avril à Octobre les lames d'eau précipitées sur le bassin.

Plutôt que calculer cette pluviosité "moyenne" à partir des courbes isohyètes (ce qui aurait nécessité le tracé d'un nombre impressionnant de cartes...) ou à partir des polygones de Thiessen, nous avons préalablement vérifié l'influence du relief, c'est-à-dire l'existence ou non d'un gradient pluviométrique (Arlery et Bediot 1972).

Une liaison pluie-altitude apparaît effectivement dans la majeure partie des cas et finalement la politique suivante a été choisie : un quadrillage du bassin a été réalisé à partir d'une carte au $1 / 200000^{\mathrm{e}}$, chaque carreau de $1 \mathrm{~cm}$ de côté (correspondant à une surface de $4 \mathrm{~km}^{2}$ ) étant affecté de son altitude moyenne (soit plus de 1100 carreaux entiers ou partiels. ..).

Dans le cas où le coefficient de corrélation pluiealtitude s'est trouvé supérieur ou égal à 0,5 , la pluviométrie moyenne à l'échelle du bassin a été calculée par la relation linéaire pluie-altitude appliquée aux données du quadrillage. Sinon les polygones de Thiessen ont été utilisés.

\subsubsection{Calcul des probabilités des précipitations estivales}

Lorsqu'on établit une prévision au 1er Juin pour le mois d'Août, on ne connaît pas les événements qui vont caractériser les mois de Juin et de Juillet.

Cependant, afin d'estimer le "débit minimum garanti" (EDF - DTG 1970) du mois d'Août, on peut introduire un "terme prévisionnel" qui, par exemple, peut être un quantile tiré de la loi de probabilité des pluies des mois de Juin et Juillet. 


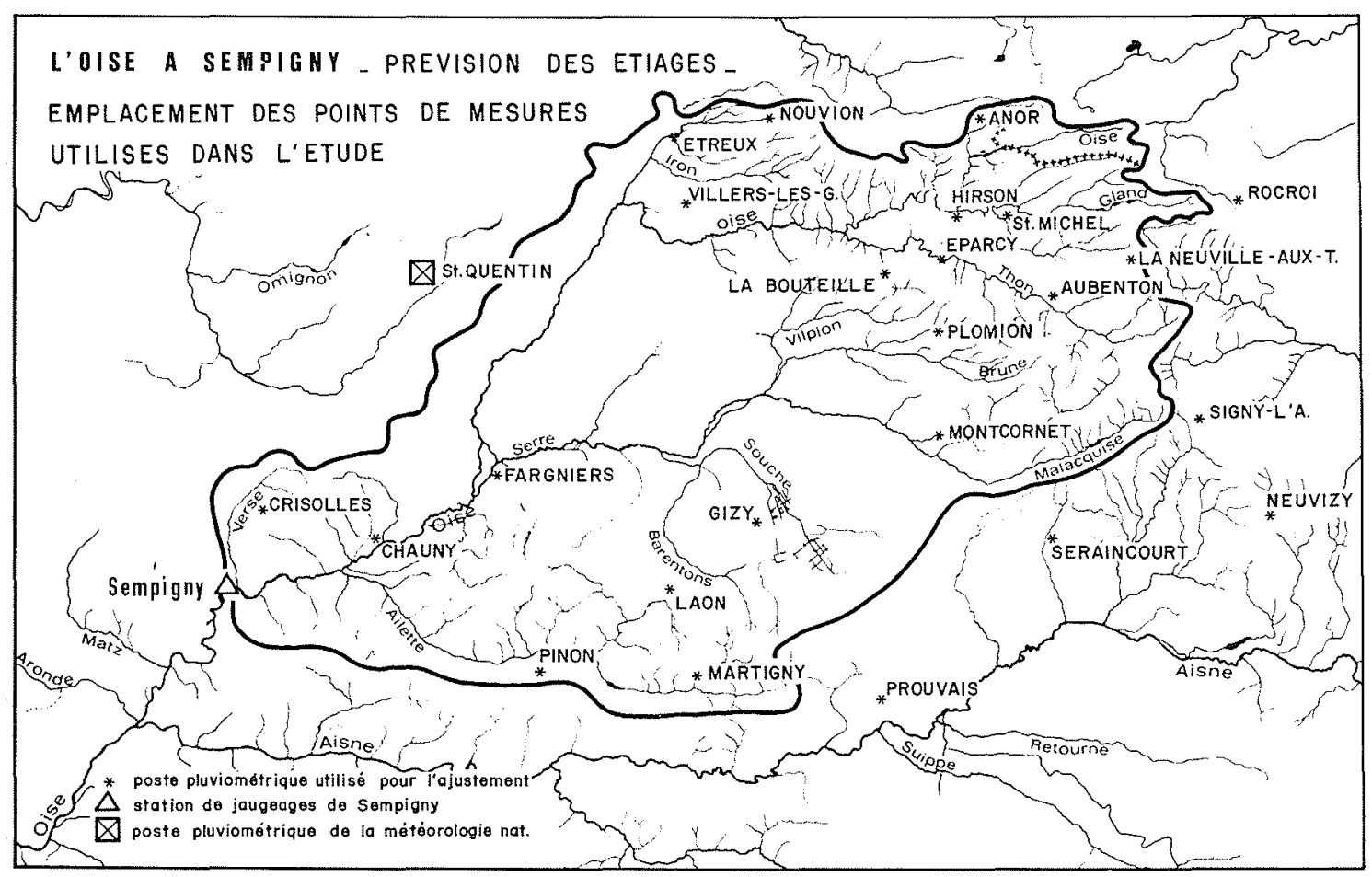

Carte 2/

Sur les lames d'eau mensuelles précipitées et sur chacun des mois de Juin à Octobre, on a procédé à l'ajustement de lois de probabilités, la plus adéquate d'entre elles étant la loi normale. Des ajustements semblables ont été effectués pour les combinaisons de 2 et 3 mois consécutifs. (Fig. 7 à 10 ).

Par exemple, si l'équation de régression retenue pour la prévision est du type :

$$
Q=\sum_{i=1}^{i=P} a_{i} X_{i}+\sum_{j=1}^{j=q} b_{j} Y_{j}+C+\epsilon
$$

où

$Q=$ variable à prévoir

$a_{i}$ et $b_{j}=$ coefficients de régression

$C=$ constante ou ordonnée à l'origine

$X_{i}=$ variable explicative connue au moment de la prévision

il y a $p$ variables $X_{i}$

$Y_{j}=$ variable explicative inconnue au moment de la prévision

il y a $q$ variables $Y_{j}$

par exemple les lames d'eau qui tomberont sur le bassin au cours de certains mois ou groupe de mois séparant la prévision de sa réalisation

$\epsilon=$ terme aléatoire que la prise en compte des variables $Y_{j}$ rend faible.

La connaissance des lois de probabilité des variables $Y_{j}$ permet de composer celles du terme $\sum_{J=1}^{J=q} b_{J} Y_{J}$ relativement facilement puisqu'il s'agit le plus souvent de variables normales indépendantes
Comme cela est répété plus loin, en associant ensuite cette loi de distribution à celle du terme aléatoire et éventuellement à celle des coefficients de régression, il est possible de prévoir non seulement la valeur de $Q$ la plus probable mais aussi d'associer une probabilité à tout intervalle de valeur concernant la réalisation de $Q$.

\subsection{Les températures}

Les valeurs des températures moyennes mensuelles employées proviennent de la station météorologique de Saint Quentin.

\subsection{La piézométrie}

Les relevés piézométriques journaliers aux stations de Beautor, Tergnier et Charmes sont récents. Ils ne seront utilisés que pour la prévision à l'aide d'un modèle pluie-débit (C'.R.E.C.).

\section{Objectifs et méthodes}

\subsection{Objectifs à atteindre}

Les objectifs de la prévision ont été choisis à priori. Ainsi, avons-nous décidé d'émettre, au cours de la première semaine d'un mois $\mathbf{J}-2$, des prévisions concernant des caractéristiques de débit pour le mcis $J$.

Les dates suivantes ont été retenues :

- début juin pour le mois d'août,

- début juillet pour le mois de septembre,

- début août pour le mois d'octobre. 
De même, pour caractériser les débits d'un mois, c'està-dire comme variable à prévoir, nous avions retenu dans un premier temps :

- $Q_{J}^{m}$, le débit moyen du mois J,

- $Q_{J}^{10}$, le débit moyen minimum de 10 jours consécutifs au cours de ce même mois $J$.

Par la suite, nous nous sommes rendus compte que de meilleures prévisions pouvaient être obtenues en utilisant :

- $Q_{J}^{5}$, le débit moyen minimum de 5 jours consécutifs du mois $J$, cette variable étant moins influencée par les pluies d'été, inconnues au moment de la prévision.

Ces caractéristiques ne renferment certainement pas la totalité de ce qu'il serait souhaitable de prévoir en ce qui concerne les bas débits. D'autres prévisions, par exemple le nombre de jours où le débit est inférieur à une certaine valeur, seraient probablement plus directement utilisables. Mais au stade exploratoire où nous sommes, il importe surtout de déterminer les possibilités de prévision en matière d'étiage, le raffinement dans le choix des variables n'intervenant que dans une seconde étape.

Les prévisions utilisent des relations ajustées sur les années d'observations antérieures, relations où entrent les réalisations de variables hydroclimatiques observées au cours des jours ou des mois qui précèdent l'émission de la prévision.

L'établissement de ces relations et le choix des variables explicatives à prendre en compte fait appel à des techniques diverses.

Nous en avons retenu un certain nombre en évitant cette fois les à priori et en adoptant une attitude expérimentale; la meilleure méthode sera celle qui nous donnera les meilleurs résultats en prévision. Nous pouvons classer les méthodes utilisées en deux catégories : celles purement stochastiques et celles comportant une partie déterministe.

\subsection{Les méthodes}

\subsubsection{Les méthodes purement stochastiques}

$\mathrm{Ce}$ sont essentiellement les régressions multiples. Leur utilisation nécessite quelques précautions sur lesquelles tout le monde s'accorde : vérification de la variance des résidus (homoscédasticité et indépendance) et de la linéarité des régressions partielles. Cependant, indépendamment de ces vérifications indispensables, des choix sont possibles qui concernent d'une part les méthodes d'ajustement et d'autre part le choix des variables explicatives à retenir.

Le calcul des coefficients de régression, c'est-à-dire de l'équation qui va servir à la prévision, peut se faire par les moindres carrés à partir de l'échantillon des observations passées. Cette technique assure par définition une variance résiduelle minimale sur cet échantillon. Mais il n'est pas certain que ce qui convient le mieux à quelques individus (l'échantillon), convienne également à d'autres indiviclus (les années où les prévisions seront émises). D'autre part, l'utilisation courante de variables explicatives non indépendantes conduit à des difficultés de calcul et à une grande variance des coefficients de régression. Si $\hat{B}$ est le vecteur de l'estimation des coefficients de régression on a :

$$
\operatorname{var}(\hat{B})=\sigma^{2} \sum_{i=1}^{k} \frac{1}{\lambda_{i}}
$$

avec :

$\sigma^{2}$ variance résiduelle de la régression

$k$ nombre de variables explicatives

$\lambda_{i}$ valeurs propres de la matrice de corrélation des variables explicatives.

Des variables explicatives corrélées conduisent à des valeurs propres presque nulles, donc à une grande variance des coefficients de régression.

De nombreuses méthodes permettant de remédier à ces défauts, ont été proposées ces dernières années. Parmi celles-ci nous avons retenu la "ridge regression" et la reǵression orthogonalisée.

Dans la "ridge regression", en s'inspirant des techniques d'identification de systèmes, on apporte une perturbation dans la diagonale de la matrice de corrélation des variables explicatives. On diminue ainsi la variance des coefficients de régression au prix d'un certain biais.

Des techniques graphiques (Hoerl et Kennard 1970) ou des algorithmes de calcul numérique (Hemmerle 1975) permettent de choisir la perturbation optimale.

La régression orthogonalisée (Marquardt 1970) se ramène à une régression classique entre la variable expliquée et un certain nombre de composantes principales tirées de la matrice de corrélation des variables explicatives.

Dans tous les cas, les ajustements obtenus par ces techniques ont, sur l'échantillon qui a servi à l'ajustement, une variance résiduelle supérieure aux moindres carrés classiques. Il est donc essentiel de conserver des observations qui ne servent pas à l'ajustement, pour pouvoir comparer ces méthodes.

Les deux méthodes ont été testées d'une part sur des échantillons tirés d'une population créée artificiellement autour d'une équation de régression multiple fixée à priori en ajoutant des variables "parasites" liées aux variables explicatives de base et d'autre part sur un exemple hydrologique avec des variables explicatives très corrélées (les pluies à différents postes d'un bassin). Dans les deux cas, les résultats en prévision ont été bien meilleurs qu'avec les moindres carrés classiques, la variance résiduelle en prévision étant dans certains cas diminuée de moitié (Debar $\mathrm{Ph}$. 1976). Mais l'efficacité de ces méthodes dépend du degré de liaison des variables explicatives.

Le choix des variables explicatives est aussi un problème qui n'est pas épuisé. Quelles que soient les méthodes, le fait de faire entrer dans l'équation de régression des variables ayant une influence importante sur les débits comme les pluies d'été (inconnues au moment de la prévision mais connues sur l'échantillon qui sert à l'ajustement des relations) permet de réduire dans des proportions importantes la variable résiduelle, donc la variance des coefficients de régression qui en dépend directement (cf. formule ci-dessus). On appelle ces variables termes prévisionnels.

En outre, en composant la loi de probabilité de la combinaison linéaire de ces variables inconnues au moment de la prévision, il est possible de prévoir non plus une valeur ponctuelle, mais un intervalle réduit où l'on a la probabilité $P$ d'observer la réalisation de l'événement prévu. 
Indépendamment de l'introduction des termes prévisionnels, les variables susceptibles d'avoir une influence sur le phénomène sont si diverses que le nombre de celles qu'on retient approche souvent le nombre d'observations et qu'il est nécessaire de faire un choix.

Une technique utilise des tests statistiques liés à l'hypothèse de normalité : tests $F$ partiels ou test $t$ de nullité des coefficients de régression; ces deux tests étant équivalents si le $F$ partiel a $I$ degré de liberté au numérateur. L'utilisation de ces tests peut se faire de manière ascendante : on rentre une variable, puis une seconde, puis une troisième. . et on teste à chaque introduction ; on peut opérer aussi de manière descendante : on prend d'abord toutes les variables et on teste ensuite celles à éliminer. Nous avons retenu la régression progressive ou méthode "Stepwise" avec, à chaque étape de la méthode ascendante, les tests de la méthode descendante (Draper et Smith 1966).

Nous avons retenu aussi une méthode que nous appellerons "Cross-Validation" (Stone M. 1974) et qui procède différemment : partant des $n$ observations constituant l'échantillon, cette méthode ajuste une régression sur $n-1$ observations, puis teste l'équation obtenue sur l'observation non prise en compte. On obtient ainsi un écart en prévision. L'observation ayant servi de test est remise dans l'échantillon, une autre est laissée de côté pour être testée en prévision et ainsi de suite jusqu'à ce que les $n$ observations aient été testées. Les variables explicatives retenues sont celles qui assurent le minimum de ce critère : moyenne des carrés des écarts en prévision. L'équation est ensuite ajustée par les moindres carrés classiques sur la totalité des observations.

\subsubsection{Les méthodes partiellement déterministes}

D'autres méthodes se distinguent des régressions car elles comportent une partie déterministe.

Ainsi, nous inspirant de la méthode de la charnière de Monsieur Roche, nous avons ajusté une exponentielle à la série des débits moyens journaliers des périodes de 3 mois considérées.

Les observations à retenir pour estimer $Q_{0}$, le débit à l'origine et la valeur de $\alpha$, la décroissance, ont été obtenues en minimisant les écarts relatifs à l'aide d'une méthode classique d'optimisation. Cette exponentielle fournit une prévision ponctuelle qu'il sera possible de transformer en un intervalle ayant la probabilité $P$ de voir se réaliser l'événement prévu, à partir de la loi de distribution des résidus. Nous comptons essayer un ajustement sur les minimums relatifs qui pourrait nous donner une limite inférieure pour la prévision.

L'étude des méthodes citées ci-dessus est déjà bien avancée et leurs résultats pourront probablement servir dès cette année pour émettre des prévisions. Nous comptons cependant les compléter dans l'avenir par d'autres méthodes à composantes déterministes.

Ainsi nous avons l'intention d'utiliser une autre méthode mi déterministe, mi stochastique : celle du débit caractéristique de Monsieur Bernier.

Nous comptons aussi utiliser un modèle déterministe conceptuel global de débits journaliers : le modèle CREC, avec les orientations suivantes:

- tout d'abord comme un moyen d'obtenir une synthèse des informations hydroclimatologiques passées, donc de l'état hydrologique du bassin au moment de la prévision, ceci par l'intermédiaire des valeurs prises par les quelques variables du modèle (essentiellement des niveaux de réservoirs qu'on essaiera d'ailleurs de relier aux niveaux piézométriques disponibles);

- ensuite comme un moyen d'obtenir une limite inférieure aux prévisions en faisant fonctionner le modèle sans pluie à partir de la date d'émission de la prévision ;

-- enfin comme un moyen de prévoir un intervalle ayant la probabilité $P$ de voir se réaliser l'événement prévu plus étroit que les intervalles précédents parce que tenant compte de la répartition des pluies joumalières sur le bassin dans l'espace et dans le temps par l'intermédiaire d'un modèle de pluie journalière décrivant cette répartition.

Cette dernière utilisation n'étant dans notre esprit qu'une étape, avant l'introduction dans le modèle de prévisions quantitatives de pluie que les météorologistes parviendront bien un jour ou l'autre à émettre.

\section{Les résultats}

Avec les méthodes de régression, les ajustements ont porté sur la période 1950-1973 (sauf 1951 et 1953), les essais de prévision sur la période 1931-1949 (Sauf 1940 et 1949).

Avec la méthode de tarissement moyen, les ajustements concernent la période 1930-1949, les essais de prévision la période 1950-1973.

\subsection{Les ajustements}

\subsubsection{Les méthodes de régression}

Un premier ajustement a été effectué en supposant connues toutes les variables, afin de vérifier si une relation linéaire simple faisant intervenir les variables température et pluie à l'échelle du bassin pouvait conduire à une reconstitution satisfaisante des caractéristiques de débit considérées (tableau 2 colonne a).

Cette étape préliminaire, fondamentale, conditionnait à elle seule la poursuite de l'étude.

Dans un deuxième temps, nous avons abandonné les débits et les températures des deux mois précédant le mois pour lequel doit être effectuée la prevision (ce délai correspondant au temps de prévision initialement fixé) mais en supposant néanmoins connue la pluviométrie sur toute la période (tableau 2 colonne b).

Enfin, le dernier ajustement a été réalisé sans introduction de terme prévisionnel et en absence de toute variable relative au délai de prévision (tableau 2 colonne c).

Il n'est pas question de faire figurer ici les quelque 108 expressions obtenues par les diverses méthodes.

Nous donnerons 2 exemples dans les tableaux suivants 3 et 4 :

- un exemple correspondant à un ajustement satisfaisant $(\rho \simeq 0,83):$ le $Q_{\text {A oût }}^{5}$, sans terme prévisionnel et avec les différentes méthodes 


\begin{tabular}{|c|c|c|c|c|c|c|c|c|c|}
\hline \multicolumn{8}{|c|}{$\begin{array}{l}\text { Variables explicatives retenues après une première sélection } \\
\text { pour la prévision du } Q_{. j}^{i} \text { de l'Oise à Sempigny }\left({ }^{1}\right)\end{array}$} & \multicolumn{2}{|c|}{ Tableau 2} \\
\hline Mois J & \multicolumn{3}{|c|}{ Août } & \multicolumn{3}{|c|}{ Septembre } & \multicolumn{3}{|c|}{ Octobre } \\
\hline $\begin{array}{l}\text { Nature de l'ajustement }\left({ }^{2}\right) \\
\text { Liste des variables }\end{array}$ & a & $\mathrm{b}$ & $c$ & a & $b$ & $\mathrm{c}$ & a & $\mathrm{b}$ & $\mathrm{c}$ \\
\hline$Q_{j-1}^{i}$ & $x$ & & & $\mathrm{x}$ & & & $x$ & & \\
\hline$Q_{i-2}^{i}$ & $\mathrm{x}$ & & & $x$ & & & $x$ & & \\
\hline$Q_{j-3}^{i}$ & $x$ & $\mathrm{x}$ & $\mathrm{x}$ & $\mathrm{x}$ & $\mathrm{x}$ & $x$ & $x$ & $x$ & $\mathrm{x}$ \\
\hline$Q_{j \sim 4}^{i}$ & & $x$ & $x$ & & $x$ & $x$ & & $x$ & $x$ \\
\hline$Q_{i-5}^{i}$ & & & & & $\mathrm{x}$ & $x$ & & $\mathrm{x}$ & $\mathrm{x}$ \\
\hline$Q_{j \cdots 6}^{i}$ & & & & & & & & $x$ & $\mathrm{x}$ \\
\hline$P_{i}^{m}$ & $x$ & $\mathrm{x}$ & & $x$ & $\mathrm{x}$ & & $\mathrm{x}$ & $x$ & \\
\hline$P_{j-1}^{m}$ & $\mathrm{x}$ & $x$ & & $\mathrm{x}$ & $\mathrm{x}$ & & $\mathrm{x}$ & $\mathrm{x}$ & \\
\hline$P_{j-2}^{m}$ & $x$ & $\mathrm{x}$ & & $\mathrm{x}$ & $x$ & & $\mathrm{x}$ & $\mathrm{x}$ & \\
\hline$P_{j-3}^{m}$ & $x$ & $x$ & & & $x$ & $x$ & & & $\mathrm{x}$ \\
\hline$P_{j \cdots 4}^{m}$ & & & & & $\mathrm{x}$ & $\mathrm{x}$ & & & $\mathrm{x}$ \\
\hline$P_{j-5}^{m}$ & & & & & & & & & $\mathrm{x}$ \\
\hline$P_{1, j-3}^{10}$ & & & $\mathrm{x}$ & & & & & & \\
\hline$P_{2, j-3}^{10}$ & & & $x$ & & & & & & \\
\hline$P_{3, j-3}^{10}$ & & & $x$ & & & & & & \\
\hline$T_{j}^{m}$ & $x$ & & & $x$ & & & $x$ & & \\
\hline$T_{j-1}^{m}$ & $\mathrm{x}$ & & & $x$ & & & $x$ & & \\
\hline$T_{j-2}^{m}$ & & & & $x$ & $x$ & & $\mathrm{x}$ & & \\
\hline$T_{j-3}$ & & $\mathrm{x}$ & $\mathrm{x}$ & & $\mathrm{x}$ & $\mathrm{x}$ & & $x$ & $\mathrm{x}$ \\
\hline$T_{j-4}$ & & $x$ & $x$ & & & $\mathrm{x}$ & & $\mathrm{x}$ & $\mathrm{x}$ \\
\hline$T_{j-5}^{m}$ & & & $x$ & & & $\mathrm{x}$ & & & $\mathrm{x}$ \\
\hline$T_{j-6}^{n}$ & & & $\mathrm{x}$ & & & $x$ & & & \\
\hline
\end{tabular}

(1) $Q_{j}^{i}$ signifie le débit du mois $j$ pour lequel on réalise la prévision.

Dans chaque cas $i$ indique que les calculs ont été faits sur la base soit des débits moyens mensuels, soit du débit minimum de 10 jours consécutifs, soit du débit minimum de 5 jours consécutifs.

(2) Les croix dans les colonnes $a, b$, c, indiquent l'emploi de la variable correspondante. Pour la signification de a, b, c, se reporter au texte 3.1.1.

- un exemple correspondant à un ajustement médiocre $(\rho \simeq 0,54)$ : le $Q_{\text {Octobre }}^{10}$, sans terme prévisionnel et avec les différentes méthodes.

Les graphiques 1 à 6 de l'annexe donnent les résultats des ajustements des débits moyens d'Août et des $Q_{\text {Aoút }}^{5}$ par la méthode Stepwise.

\subsubsection{Remarques sur les ajustements par méthodes de régression}

\section{a) Remarques d'ordre hydrologique}

Le $Q_{\text {Août }}^{5}$ et, à un degré moindre, le $Q_{\text {Aaût }}^{10}$ sont davantage représentatifs du phénomène général de tarissement que le débit moyen du mois d'Août. Ce dernier subit l'influence des crues brèves liées le plus souvent à des phé- nomènes orageux localisés. La prévision du débit minimum garanti du mois d'Août est meilleure sur la base du débit minimum de 5 jours consécutifs : c'est inattendu mais ça s'explique bien hydrologiquement.

Au fur et à mesure que l'on avance dans la saison, le phénomène inverse se produit : on obtient par exemple une meilleure reconstitution du débit moyen d'Octobre que du $Q_{\text {Octobre }}^{10}$.

Avec la Stepwise, la principale variable explicative du $Q_{\text {Août }}^{5}$ est le $Q_{\text {Mai }}^{5}$. En revanche, dans le cas du débit moyen d'Octobre (ou du $Q_{\text {Octobre }}^{10}$ ), plus aucun terme débit n'apparaît comme variable explicative : seule la pluviosité de Juin et Juillet intervient. Le phénomène d'autocorrélation est alors remplacé par un antécédent pluviométrique lié grossièrement au rendement $\mathrm{du}$ bassin qui augmente au 


\begin{tabular}{|c|c|}
\hline \multicolumn{2}{|c|}{$\begin{array}{l}\text { Résultats des ajustements par les méthodes de régression } \\
\text { Exemple } n^{\circ} 1: \text { Calcul du } Q_{\text {Aoút avec } 9 \text { variables explica- }}^{5} \\
\text { tives, connues au 1er Juin. }\end{array}$} \\
\hline Stepwise $(\rho=0,83)$ & $Q_{\text {Août }}^{5}=0,329 Q_{\mathrm{Mai}}^{5}+0,04 P_{\mathrm{Mai}}+1,991$ \\
\hline Cross-Validation $(\rho=0,83)$ & $Q_{\text {Août }}^{5}=0,329 Q_{\mathrm{Mai}}^{5}+0,04 P_{\mathrm{Mai}}+1,991$ \\
\hline $\begin{array}{l}\text { Regression orthogonalisée } \\
\text { (composantes principales) } \\
\qquad \rho=0,84\end{array}$ & $\begin{aligned} Q_{\text {Aoút }}^{5} & =0,1315 Q_{\text {Avril }}^{5}+0,1853 Q_{\text {Mai }}^{J}+0,076 P_{1(\text { Mai })}^{10} \\
& +0,072 P_{(\mathrm{Mai})}^{10}+0,058 P_{(\mathrm{Mai})}^{10}-0,246 T_{\text {Février }} \\
& +0,414 T_{\text {Mars }}+0,420 T_{\text {Avril }}-0,012 T_{\text {Mai }}-5,69\end{aligned}$ \\
\hline $\begin{array}{l}\text { Ridge regression } \\
\qquad \rho=0,87\end{array}$ & $\begin{aligned} Q_{\text {Août }}^{5}= & -0,192 Q_{\text {Avril }}^{5}+0,522 Q_{\text {Mai }}^{5}+0,023 P_{1 \text { (Mai) }}^{10} \\
& +0,041 P_{3(\text { Mai) }}^{10}-0,22 T_{\text {Tévrier }}+0,237 T_{\text {Mars }} \\
& +0,918 T_{\text {Avril }}+0,129 T_{\text {Mai }}-6,071\end{aligned}$ \\
\hline $\begin{array}{r}\text { Moindres carrés } \\
\qquad \rho=0,89\end{array}$ & $\begin{aligned} Q_{\text {Août }}^{5} & =-0,265 Q_{\text {Avril }}^{5}+0,721 Q_{\text {Mai }}^{5}+0,032 P_{1 \text { (Mai) }}^{10} \\
& +0,056 P_{3(\text { Mai) }}^{10}-0,303 T_{\text {Février }}+0,328 T_{\text {Mars }} \\
& +1,267 T_{\text {Avril }}+0,178 T_{\text {Mai }}-13,10\end{aligned}$ \\
\hline
\end{tabular}

\begin{tabular}{|c|c|}
\hline \multicolumn{2}{|c|}{$\begin{array}{l}\text { Résultats des ajustements par les méthodes de régression } \\
\text { Exemple } n^{\circ} 2: \text { Calcul du } Q_{\text {Octobre }}^{10} \text { avec } 9 \text { variables expli- } \\
\text { catives, connues au } 1^{\text {er }} \text { Août. }\end{array}$} \\
\hline Stepwise $\rho=0,54$ & $Q_{\text {Octobre }}^{10}=0,108\left(P_{\text {Juin }}+P_{\text {Juillet }}\right)+2,087$ \\
\hline Cross-Validation $\rho=0,54$ & $Q_{\text {Octobre }}^{10}=0,108\left(P_{\text {Juin }}+P_{\text {Juillet }}\right)+2,087$ \\
\hline $\begin{array}{l}\text { Regression orthogonalisée } \\
\text { (composantes principales) } \\
\qquad \rho=0,71\end{array}$ & $\begin{aligned} Q_{\text {Octobre }}^{10}= & -0,0194 Q_{\text {Avril }}^{10}-0,0305 Q_{\text {Mai }}^{10}+0,1276 Q_{\mathrm{Juin}}^{10}+0,2109 Q_{\mathrm{Juillet}}^{10} \\
& -0,3729 T_{\mathrm{Mai}}-0,9201 T_{\mathrm{Juin}}-0,4215 T_{\text {Juillet }}-0,0779 P_{\mathrm{Mai}} \\
& +0,0892\left(P_{\mathrm{Juin}}+P_{\mathrm{Juillet}}\right)+31,495\end{aligned}$ \\
\hline $\begin{array}{l}\text { Ridge regression } \\
\qquad \rho=0,72\end{array}$ & $\begin{aligned} Q_{\text {Octobre }}^{10}= & -0,253 Q_{\mathrm{Avril}}^{10}+0,260 Q_{\mathrm{Mai}}^{10}-0,336 Q_{\mathrm{Juin}}^{10}+0,577 Q_{\mathrm{Juillet}}^{10} \\
& -0,329 T_{\mathrm{Mai}}-0,977 T_{\mathrm{Ju} i n}+0,118 T_{\mathrm{Juillet}}-0,064 P_{\mathrm{Mai}} \\
& +0,016\left(P_{\mathrm{Juin}}+P_{\mathrm{Juillet}}\right)+34,303\end{aligned}$ \\
\hline $\begin{array}{l}\text { Moindres carrés } \\
\qquad \rho=0,74\end{array}$ & $\begin{aligned} Q_{\text {Octobre }}^{10}= & -0,540 Q_{\text {Avril }}^{10}+0,555 Q_{\text {Mai }}^{10}-0,716 Q_{\mathrm{Juin}}^{10}+1,233 Q_{\mathrm{Juillet}}^{10} \\
& -0,702 T_{\mathrm{Mai}}-2,086 T_{\mathrm{Juin}}+0,253 T_{\text {Juillet }}-0,137 P_{\mathrm{Mai}} \\
& +0,035\left(P_{\mathrm{Juin}}+P_{\mathrm{Juillet}}\right)+55,237\end{aligned}$ \\
\hline
\end{tabular}

fur et à mesure que les températures (ou pouvoir évaporant) de l'air diminuent.

\section{b) Remarque d'ordre statistique}

Les essais effectués ont uniquement considéré des valeurs naturelles et il semble important de reprendre ultérieurement ces ajustements en vérifiant davantage la linéarité des régressions partielles et en les corrigeant éventuellement par des changements de variables (log des débits).

\subsubsection{Choix d'une exponentielle moyenne}

Sur la période 1930-1949, nous avons ajusté une exponentielle "moyenne" de formule :
$Q_{j}=Q_{0} e^{-\alpha} j$ où $j$ correspond à l'indice du jour compté à partir du départ de la prévision ( $1^{\text {er }}$ Juin, $1^{\text {er }}$ Juillet).

Cet ajustement, réalisé par minimisation d'un écart relatif, a conduit aux valeurs suivantes de $Q_{0}$ et de $\alpha$.

\begin{tabular}{|c|c|c|}
\hline & $Q_{0}$ & $\alpha$ \\
\hline 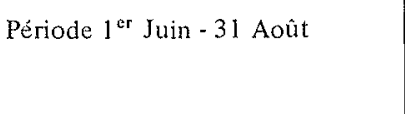 & $\begin{array}{c}\text { Débit moyen journalier } \\
\text { sur } 14 \text { jours } \\
\text { avant le } 1^{\text {er }} \text { Juin }\end{array}$ & 0,0103 \\
\hline Période $1^{\text {er Juillet - } 30 \text { Septembre }}$ & $\begin{array}{c}\text { Débit moyen journalier } \\
\text { sur } 6 \text { jours } \\
\text { avant le } 1^{\text {er Juillet }}\end{array}$ & 0,0103 \\
\hline
\end{tabular}




\subsection{Prévision}

La validité des formulations obtenues et le choix de l'une d'entre elles ne peut se faire qu'après avoir montré leur adéquation sur une série d'informations hors période d'ajustement.

\subsubsection{Prévision par les méthodes de régression}

Nous présenterons certains résultats obtenus sur la période 1931-1949. Il parait important de souligner que l'information pluviométrique sur cette période est nettement plus réduite (entre 6 et 7 postes suivant l'année) et que cette mauvaise connaissance conditionne nécessairement la qualité des résultats.

Les expressions concernant le $Q^{5}$ du mois d'Août, le $Q$ moyen du mois de Septembre et le $Q$ moyen du mois d'Octobre ont été testées sur l'ensemble des méthodes. avec et sans terme prévisionnel. La figure 6 montre les résultats obtenus pour le $Q^{5}$ d'Août sans termes prévisionnels.

La somme des carrés des écarts $\left(Q_{i}^{i} \text { obs }-Q_{j}^{i} \text { prévu }\right)^{2}$ a été calculée pour chaque méthode et les résultats, classés par ordre croissant, ont été portés graphiquement (figures $n^{\circ} 11,12,13$ ).

Remarque 1. Il ne semble pas possible de proposer une véritable hiérarchie des méthodes, telle méthode n'étant pas systématiquement meilleure que telle autre. On peut néanmoins remarquer la régularité des performances de la régression orthogonalisée. (Fig. 11, 12, 13).

Remarque 2. La médiocrité des résultats obtenus par introduction d'un terme prévisionnel avec la méthode Stepwise pour la prévision des $Q^{5}$ du mois d'Août, peut surprendre. Un examen des valeurs prévues montre que sur la période 1942-1949, quatre valeurs observées sur huit sont en-dessous de l'intervalle de confiance $(0,1$ 0,9 ). L'équation correspondante s'écrit :
$Q_{\text {Août }}^{5}$ prévu $=0.3321 Q_{\mathrm{Mai}}^{5}+0.0373 \mathrm{P}_{\mathrm{Mai}}+0.7471 T_{\text {Avril }}^{0}$

+ Terme prévisionnel - 11,03

Compte tenu de la forte valeur du coefficient de la température d'Avril, il suffit d'un écart de $3^{\circ} \mathrm{C}$ pour que la valeur de débit soit modifiée de plus de $2 \mathrm{~m}^{3} / \mathrm{s}$. Comme l'intervalle de confiance correspond à $\pm 2,5 \mathrm{~m}^{3} / \mathrm{s}$ autour de la moyenne, on voit le rôle fondamental joué par la variable température.

Les valeurs de température d'Avril à Saint-Quentin pour la période 1942-1949 étant anormalement élevées (toutes $\geqslant 9,8^{\circ} \mathrm{C}$ ), nous nous retrouvons avec une série de températures de moyenne forte $\left(10,7^{\circ} \mathrm{C}\right)$ et d'écarttype faible $\left(0,48^{\circ} \mathrm{C}\right)$. La série de température sur la période d'ajustement est d'une toute autre forme, puisque sa moyenne est de $8,87^{\circ} \mathrm{C}$ et son écart-type de $1,36^{\circ} \mathrm{C}$.

Cette remarque sur la température pour la période 1942-1949 est générale à l'ensemble des relevés des stations voisines (Lille, Reims, Abbeville) et ne peut être rattachée à un comportement isolé de la station de Saint-Quentin.

Par ailleurs, il n'existe sur la période d'ajustement pas de lien (coefficients de corrélation $<0,25$ ) entre la température du mois d'Avril et les températures ou les pluies des mois suivants.

Ceci doit inciter à une grande prudence. A la limite, il semblerait préférable non pas de choisir une période plus ou moins courte d'ajustement pour laquelle la pluviosité est bien connue, mais de faire une impasse sur la pluie en ne considérant qu'une information réduite autorisant une plus longue série de débits et donc permettant plusieurs ajustements.

Cette façon de faire devrait permettre d'éliminer le type de problème rencontré avec la température sur l'échantillon que nous avons considéré.

Ainsi, en utilisant comme période d'ajustement non plus 1952-1973, mais 1931-1973, la méthode Stepwise

\begin{tabular}{|c|c|c|c|c|c|c|c|c|c|c|c|}
\hline \multicolumn{10}{|c|}{$\begin{array}{l}\text { Bilan de la comparaison en tre valeurs observées } \\
\text { et valeurs prévues du } Q^{5} \text { aout }\end{array}$} & \multicolumn{2}{|c|}{ Tableau 5} \\
\hline \multicolumn{10}{|c|}{ Sans terme prévisionnel } & \multicolumn{2}{|c|}{$\begin{array}{c}\text { Avec terme } \\
\text { prévisionnel } \\
\mathrm{P}_{0}=0,5\end{array}$} \\
\hline $\begin{array}{l}\text { Date } \\
\text { des } \\
\text { relevés }\end{array}$ & $\begin{array}{l}\stackrel{\infty}{:} \\
\stackrel{0}{0} \\
0 \\
0\end{array}$ & $\frac{0}{\overline{0}}$ & 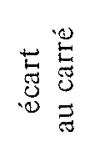 & $\frac{\omega}{\tilde{J}}$ & 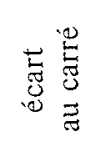 & $\frac{\ddot{0}}{\tilde{J}}$ & $\begin{array}{l}\text { 哥 } \\
\text { 总 } \\
\text { 总 }\end{array}$ & 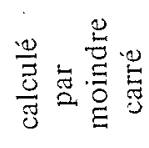 & 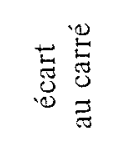 & 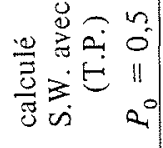 & 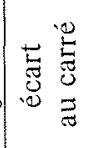 \\
\hline 1942 & 12.2 & 12,2 & 0 & 15,9 & 13,69 & 15.25 & 9,3 & 16,75 & 20,7 & 13.6 & 1,96 \\
\hline 1943 & 8,0 & 9,0 & 1,0 & 13,25 & 27,5 & 9,95 & 3,8 & 13,26 & 27,66 & 11,3 & 10,89 \\
\hline 1944 & 5,8 & 5,2 & 0,36 & 8,14 & 5,47 & 4,03 & 3,1 & 6,14 & 0,11 & 6,66 & 0,74 \\
\hline 1945 & 9,1 & 12,4 & 10,89 & 14,74 & 31,8 & 13,6 & 20,25 & 15,18 & 36,96 & 14 & 24 \\
\hline 1946 & 9,5 & 11,7 & 4,84 & 13,25 & 14,06 & 10,86 & 1,84 & 13,12 & 13,1 & 11,03 & 2,3 \\
\hline 1947 & 8,7 & 10 & 1,69 & 11,98 & 10,76 & 12,27 & 12,74 & 11,7 & 9,0 & 11,56 & 8,18 \\
\hline 1948 & 15,5 & 9,3 & 38,44 & 11,68 & 14,59 & 10,4 & 26,1 & 11,08 & 19,53 & 10,4 & 26 \\
\hline 1949 & 6,1 & 9,0 & 8,41 & 11,4 & 28,09 & 8,06 & 3,84 & 10,76 & 21,7 & 10,8 & 22 \\
\hline \multicolumn{3}{|c|}{$\Sigma$ des écarts } & 64,63 & & 145,9 & & 80,9 & & 148.76 & & 96,1 \\
\hline
\end{tabular}



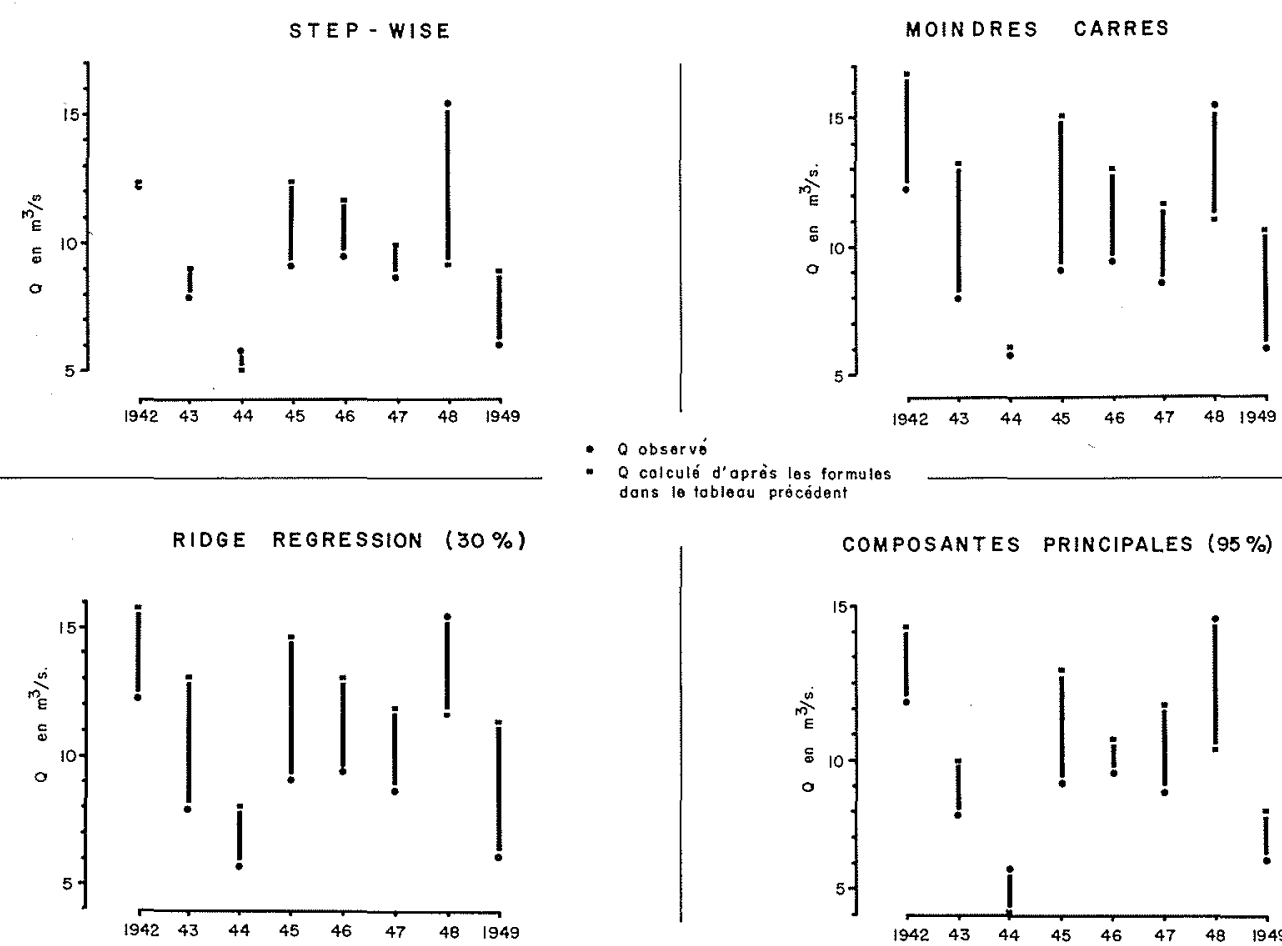

COMPOSANTES PRINCIPALES (95\%)

- Q calculé d'apròs los formules

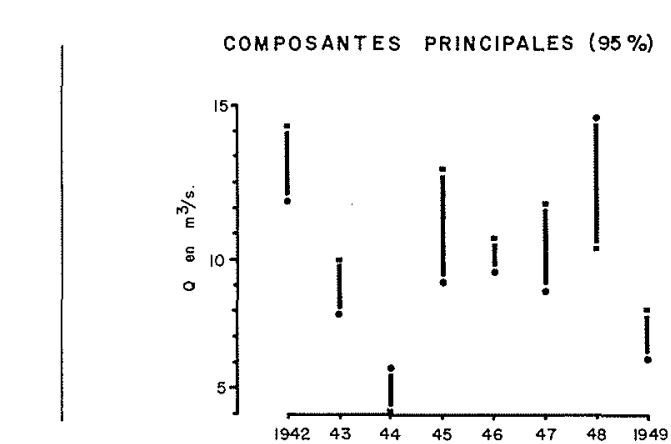

$1 /$ L'Oise à Sempigny. Prévision au $1^{\text {er }}$ Juin du $Q_{5}$ Août (pas de terme prévisionnel).

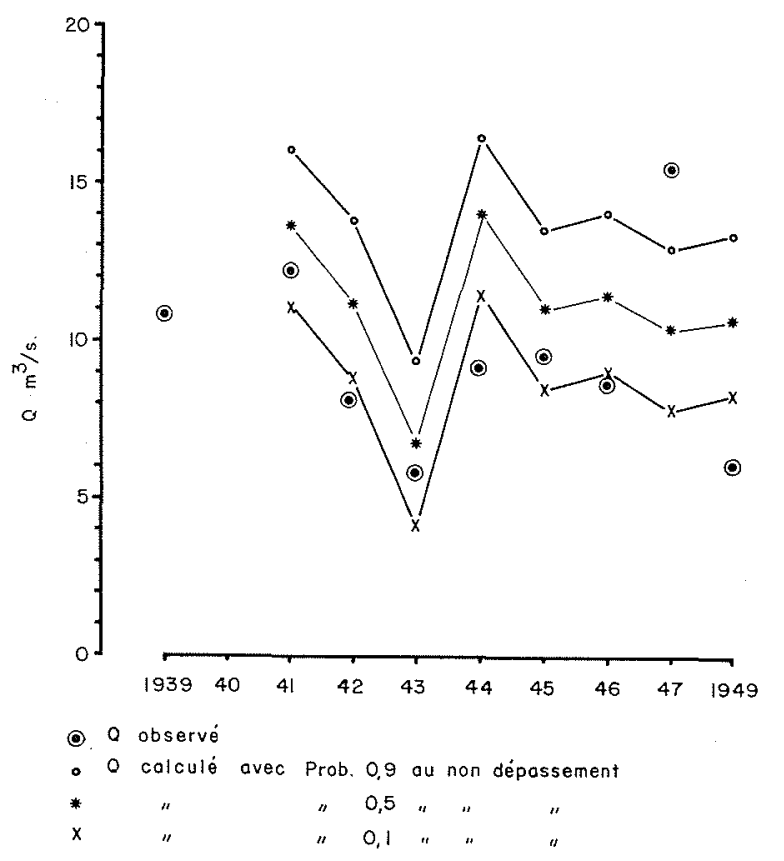

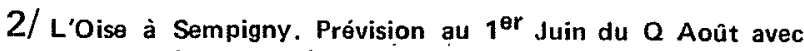
terme prévisionnel. ( $P$ Juin $+P$ Juillet), par la méthode

\section{Step-Wise}

pour le $Q^{5}$ du mois d'Août, avec terme prévisionnel, conduit à l'équation :

$$
Q_{\text {Aout }}^{5} \text { prévu }=0,309 Q_{\text {Mai }}^{5}+\text { Terme prévisionnel }-3,55,
$$

dans laquelle le terme température d'Avril n'apparait plus :
Remarque 3. L'influence des pluies qui se produisent entre l'émission de la prévision et sa réalisation est schématisée par la largeur de l'intervalle de confiance 0,1 - 0,9 sur les figures 14, 15, 16. Faible pour le $Q^{5}$ du mois d'Août, cet intervalle augmente considérablement pour le débit moyen de Septembre et encore plus pour le débit moyen d'Octobre. Ces 2 dernières variables ne pourront guère être prévues que sur le plan d'un débit minimum ayant une certaine probabilité de ne pas être atteint.

\subsubsection{Prévision de la méthode de l'exponentielle moyenne}

Le calage des paramètres $Q_{0}$ et $\alpha$ ayant été réalisé sur la période 1930-1949, il n'était pas question de comparer les résultats obtenus sur cette période d'ajustement avec ceux obtenus par les autres méthodes. Ce sera fait ultérieurement.

L'extrapolation a été effectuee sur la période 1950-1973. Nous avons fait figurer les $Q_{\text {Août }}^{5}$ et les $Q_{\text {Août obtenus par }}^{10}$ l'exponentielle issue du $1^{\text {er }}$ Juin (Les valeurs de $Q^{5}$ et $Q_{\text {Septembre }}^{10}$ obtenues par l'exponentielle issue du $1^{\text {er }}$ Juillet, nettement plus faibles que les valeurs réelles, ne sont pas présentées ici). Les fortes valeurs de $Q_{\text {Août }}^{5}$ et

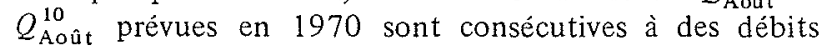
élevés de Mai (le débit moyen de ce mois est de $77,5 \mathrm{~m}^{3} / \mathrm{s}$ alors que la moyenne de référence est de $36 \mathrm{~m}^{3} / \mathrm{s}$ ).

La décrue de la période Juin-Juillet correspond à la vidange du champ d'inondation (stockage de surface puis nappe alluviale) sans qu'aucune recharge pluviométrique importante intervienne. 


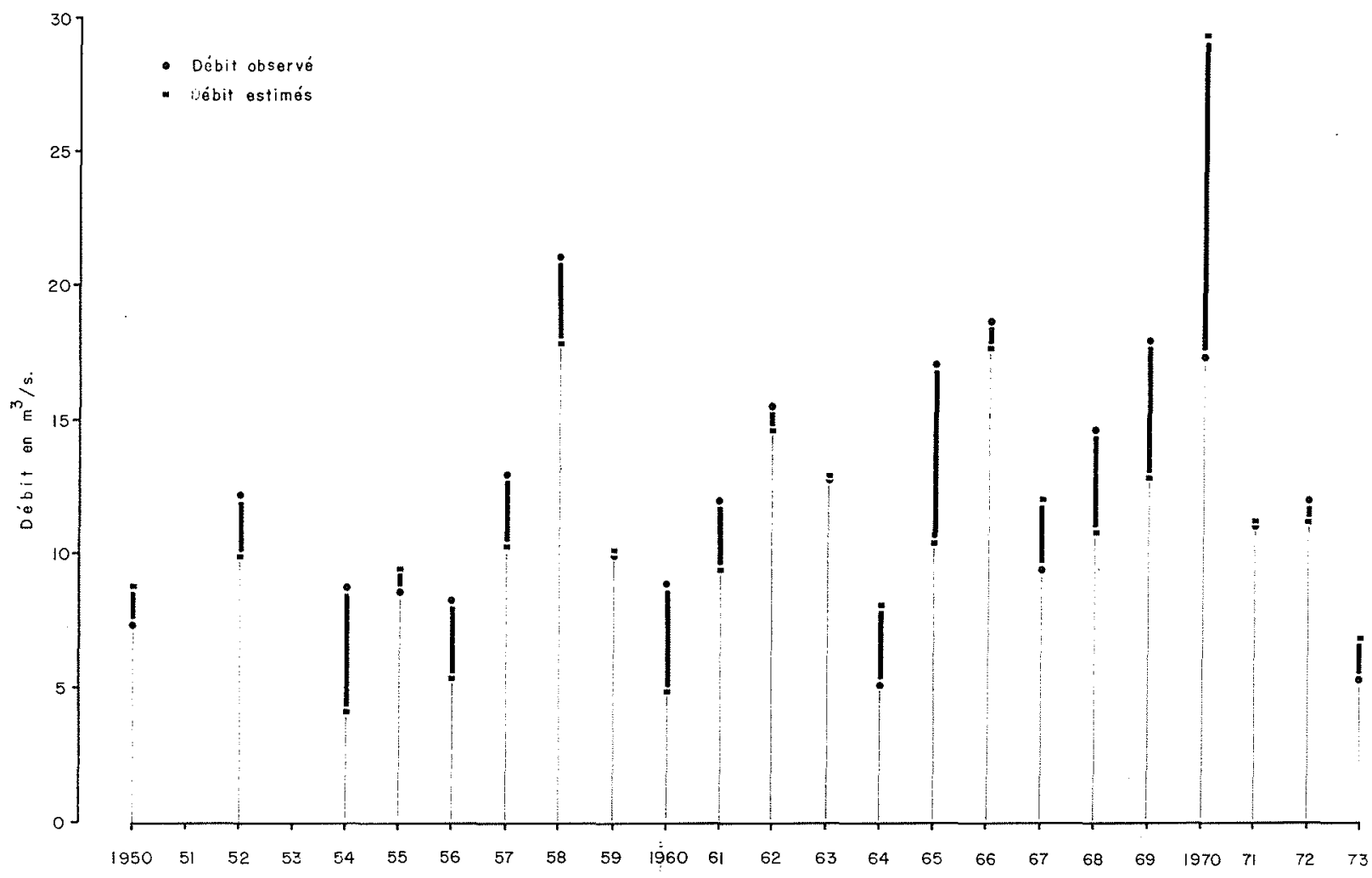

3/ L'Oise à Sempigny. Ecart entre les $O_{5}$ du mois d'Aout observés et estimés par une exponentielle ajustée aux débits moyens journaliers des 20 années précédentes.

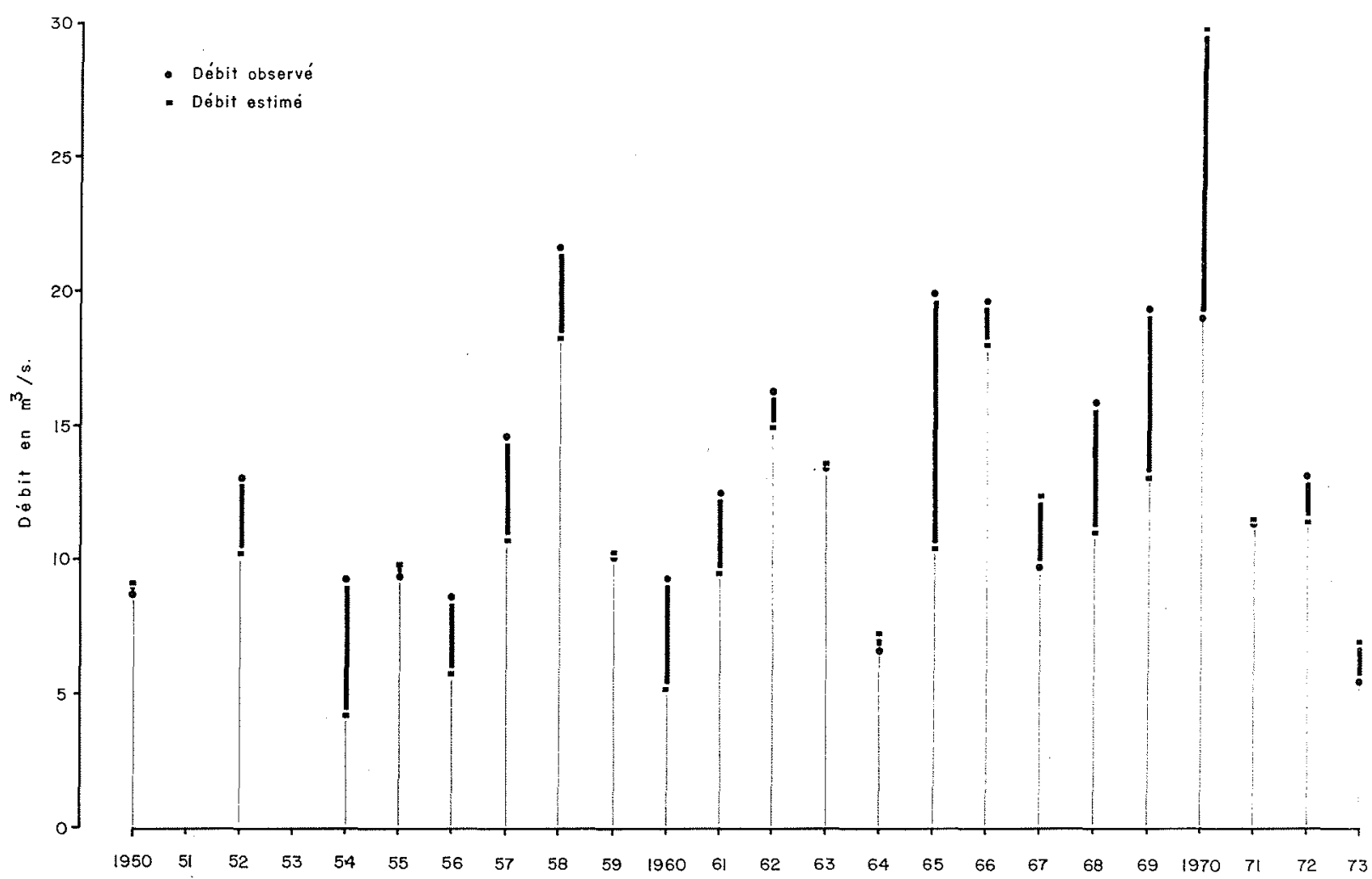

4/ L'Oisa à Sempigny. Ecart entre les $Q$ du mois d'Août observés et estimés par une exponentielle ajustée aux débits moyens journaliers des $\mathbf{2 0}$ années précédentes. 


\section{Conclusion}

Ces premiers résultats obtenus sur l'Oise à Sempigny sont encourageants : il est possible de prévoir un débit minimum garanti deux mois à l'avance. L'adjonction d'un "terme prévisionnel" est une première étape qui permet d'afficher une probabilité de réalisation.

Il reste encore diverses possibilités d'affinement avec :

- l'introduction de nouvelles variables explicatives (niveau des nappes par exemple);

- l'emploi d'autres termes prévisionnels (par exemple la température) ;

- l'extension des recherches à des bassins voisins.

Par ailleurs, la mise en cuvre d'une prévision météorologique des quantités de pluies à longue échéance (1 à plusieurs mois) demeure, à ce jour, la seule issue pour la prévision efficace de la durée, donc du degré de sévérité, des étiages.

Dès le début Juin 1976, on émettra la prévision du débit minimum garanti de 5 jours consécutifs du mois d'Août suivant. Des difficultés vont surgir pour assurer la récolte des données nécessaires dans le plus bref délai. $\mathrm{Si}$, malgré toutes ses imperfections, cette prévision s'avère économiquement utile pour la gestion de l'eau, ces difficultés trouveront vite une solution.

\section{Bibliographie}

ARLERY (R.), BEDIOT (G.), TIXERONT (J.) (1972). - Les bassins de la Seine et des cours d'eau normands. Tome 1 . Ressources d'eau et données hydrologiques - fascicule 3 données climatiques -112 pages. (AFBSN).

BEDIOT (G.), RENARD (D.) (1975) - Seine-Normandie, eaux superficielles; volume $B$ données hydrométriques du fichie de bassin - fascicule 2 caractéristiques des bassins versants. Jeaugeages occasionnels et releves limnimétriques - Cahiers techniques $\mathrm{n}^{\circ} 2-179$ pages. (AFBSN).

BERNIER (1963) - "La prévision statistique des bas débits". Compte rendu du Congrès de Berkeley A.I.H.S. n 63 - page 340-351.

CORMARY -GUILBOT (1971) - Etude générale de quelques modèles déterministes, Laboratoire u'Hydrologie, Montpellier EDF/LNH. Rapport D.G.R.S.T. n 6 ronéotypé - 16 pages 13 planches.

DEBAR (PH.) (1976) - Programmation et expérimentation de méthodes d'estimation d'équations de régression multiples en vue de prévision. Laboratoire d'Hydrologie Mathématique. U.S.T.L. Montpellier.

DRAPER et SMITH (1966) - Applied Regression Analyses. John Wiley and sons Inc
EDF Division technique générale (1970) - Prévision d'apports de la Loire à Gien en période d'étiage (Juin à Octobre). 6 pages + note complémentaire avec nombreux tableaux et graphiques.

Guilbor (A.), Avalos-Lingan (R.) (1975) - Prévision des étiages sur le bassin de l'Oise à Sempigny 1. Examen des données pluviométriques disponibles - 110 pages.

HEMMERLE (W.J.) (1975) - An explicit solution for generalized Ridge Technometrics - Volume $17 \mathrm{n}^{\circ} 3$ - page 309-314.

HOERL et KENNARD (1970) - Ridge Regression : Biased estimation for non Orthogonal problems - Technometrics Volume $12 \mathrm{n}^{\circ} 1$ page 55-82.

LAVABRE (J.), MASSON (J.M.), SAURIN (J.) (1974) - Etude hydrologique à la prise du réservoir d'Origny Sainte Benoite 46 pages. (AFBSN).

MARQUARDT (D.W.) (1970) - Generalized Inverses, Ridge Regression, Biased Linear Estimation And Nonlinear Equation Technometrics Volume $12 n^{\circ} 3$ - page 591-612.

RochE (M.) - Hydrologie de surface - Gauthier Villars - Paris Collection ORSTOM.

Service des forces hydrauliques - $1^{\text {er }}$ Circonscription Electrique (1960). Débits de l'Oise en amont du confluent avec l'Aisne Stations de Plessis Brion et de Sempigny - Etude $n^{\circ} 2-7$ pages + tableau et graphiques.

Service Spécial de la Navigation Belgique Paris Est (?). Mesures de débits et jaugeages : l'Oise à Sempigny.

STONE (M.) (1974) - Cross Validation choice and Assesment of statistical predictions - Applied statistics Volume 2 page 111 143.

\section{Annexe}

\section{A. Exemples graphiques}

Figures 1 à 6 : Résultats d'ajustements par la méthode Stepwise.

Figures 7 à 10 : Ajustements d'une loi normale aux précipitations estivales.

Figures 11 à 13 : critères d'écarts obtenus par les différentes méthodes.

Figures 14 à 16 : Résultats en prévision, avec intervalles de confiance.

Figure 17 : Exemple de tracé de l'exponentielle moyenne.

\section{B. Tableau}

$\mathrm{N}^{\circ} 1$ : Les températures à Saint Quentin. 


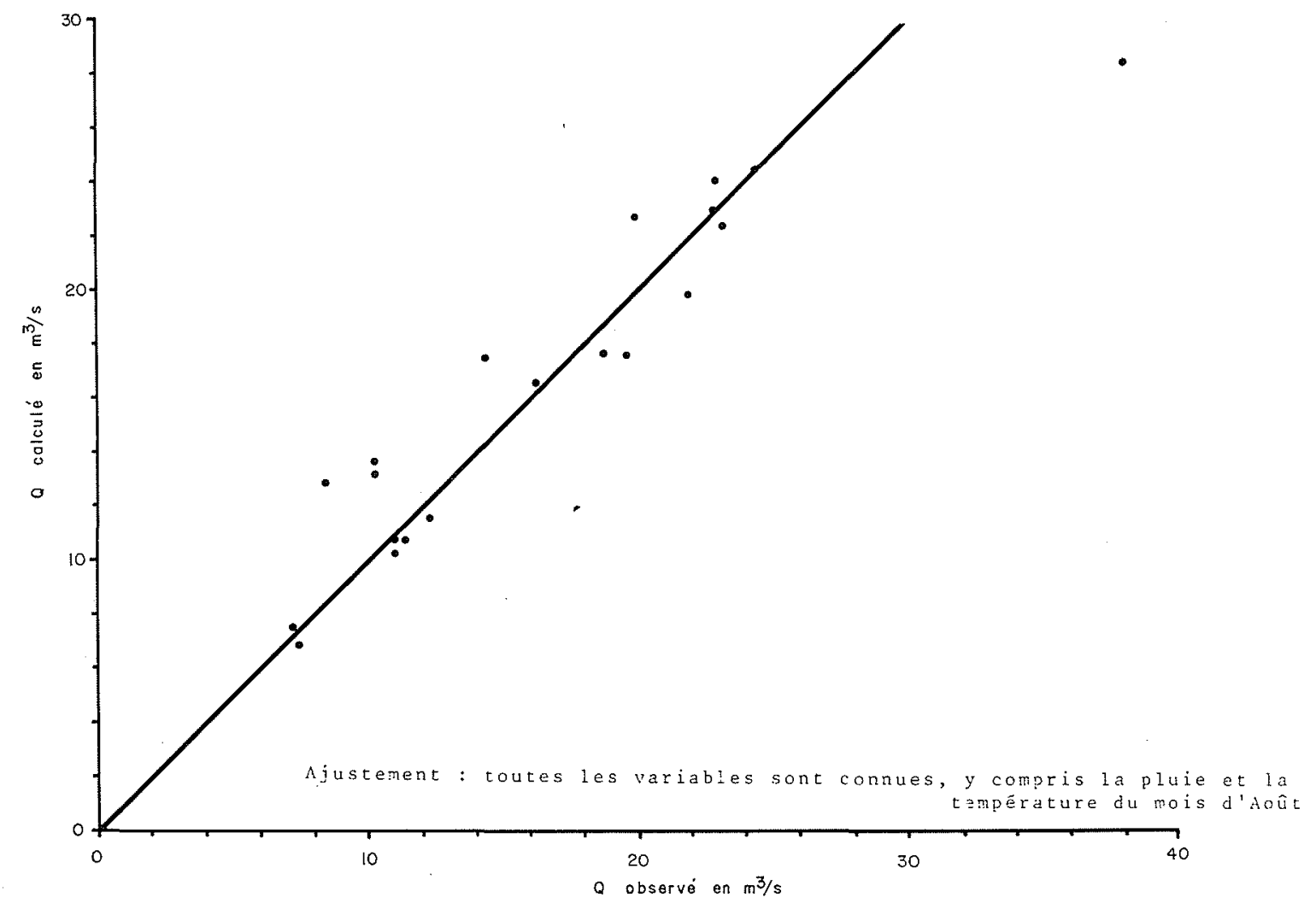

1/ L'Oise à Sempigny. Calcul du débit moyen au mois d'Août. Méthode de régression progressive Step-Wise.

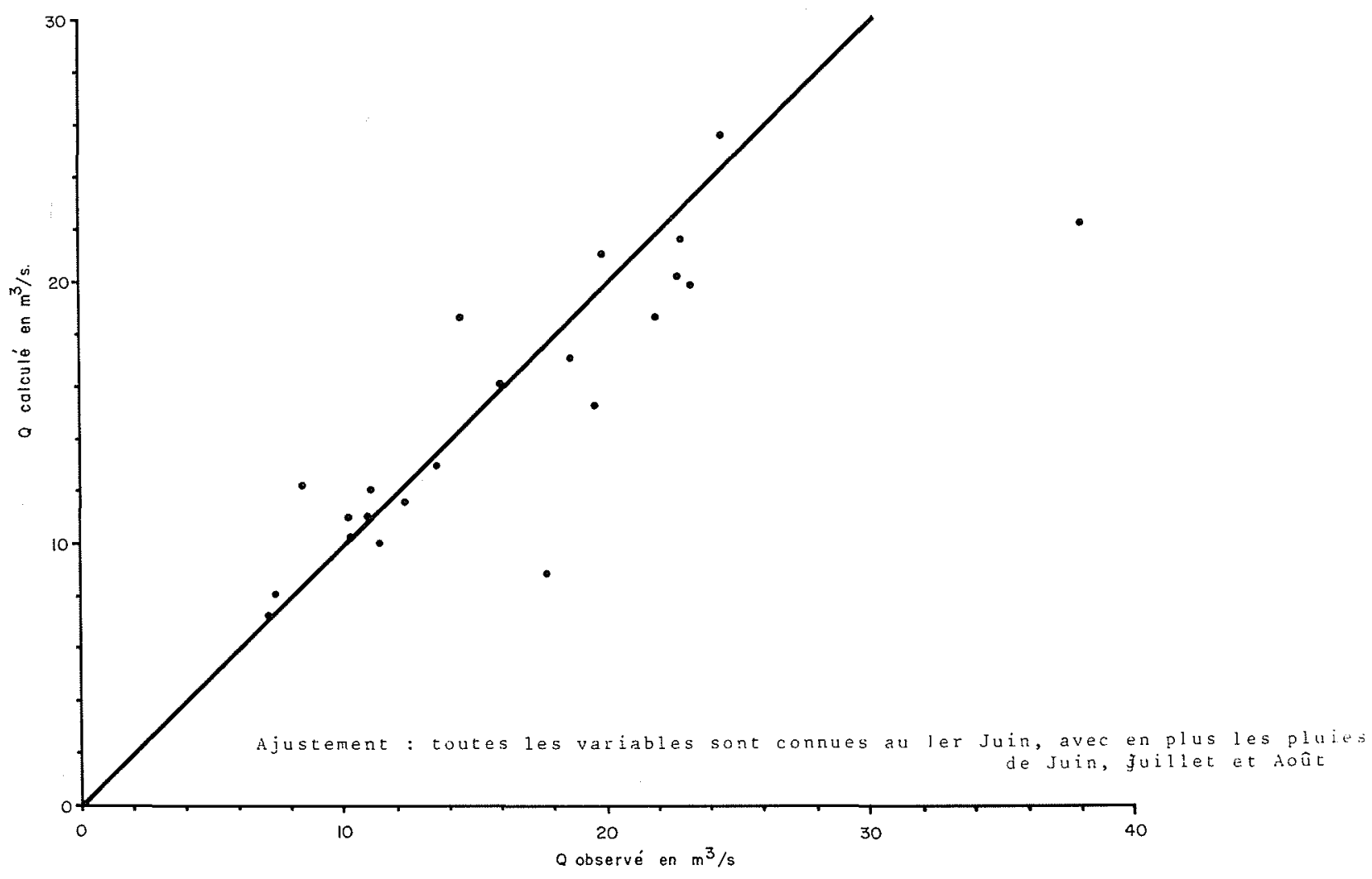

2/ L'Oise à Sempigny. Calcul du débit moyen au mois d'Août. Méthode de régression progressive Step-Wise 


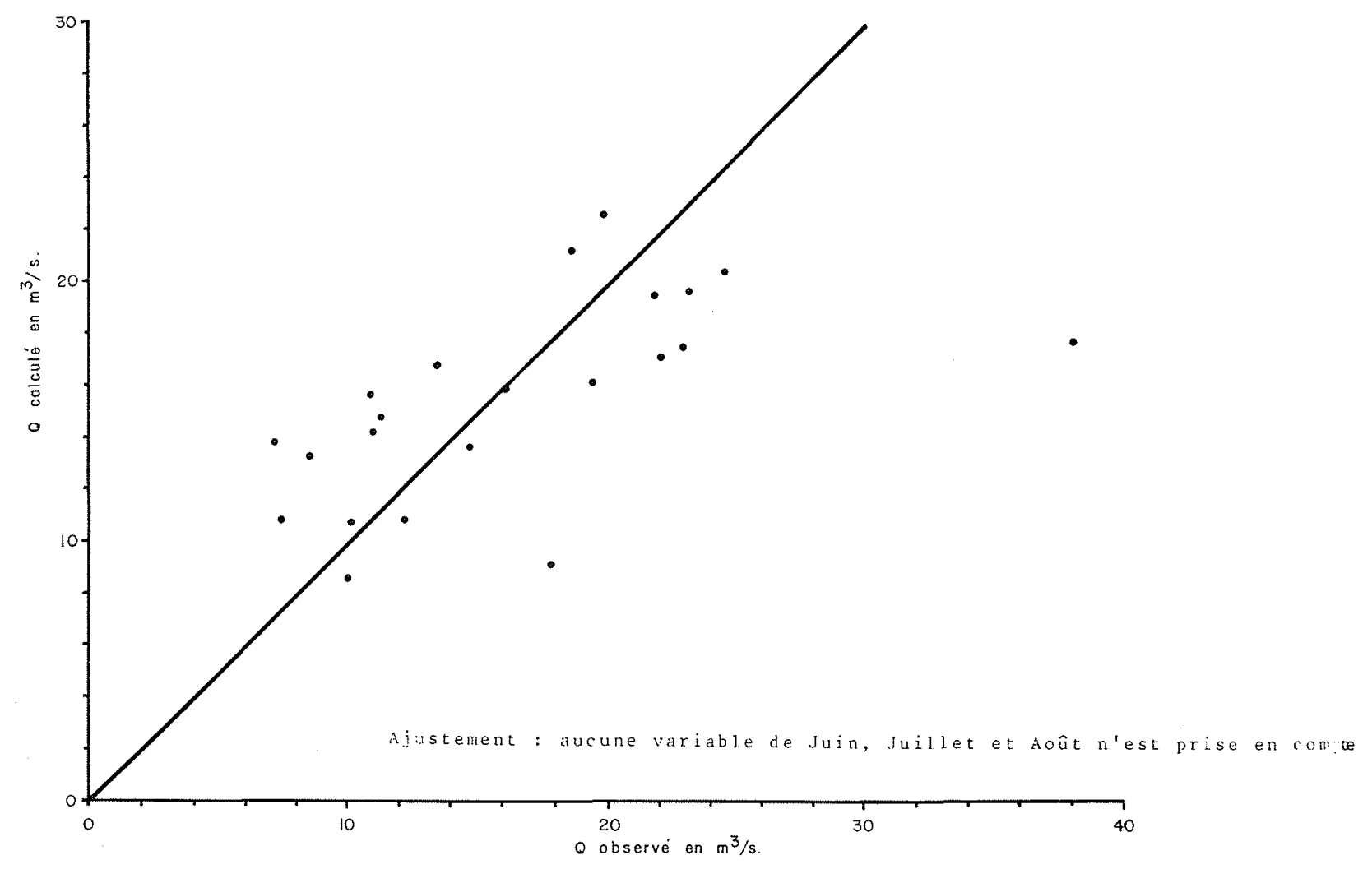

3/L'Oise à Sempigny. Calcul du débit moyen au mois d'Août. Méthode de régression progressive Step-Wise.

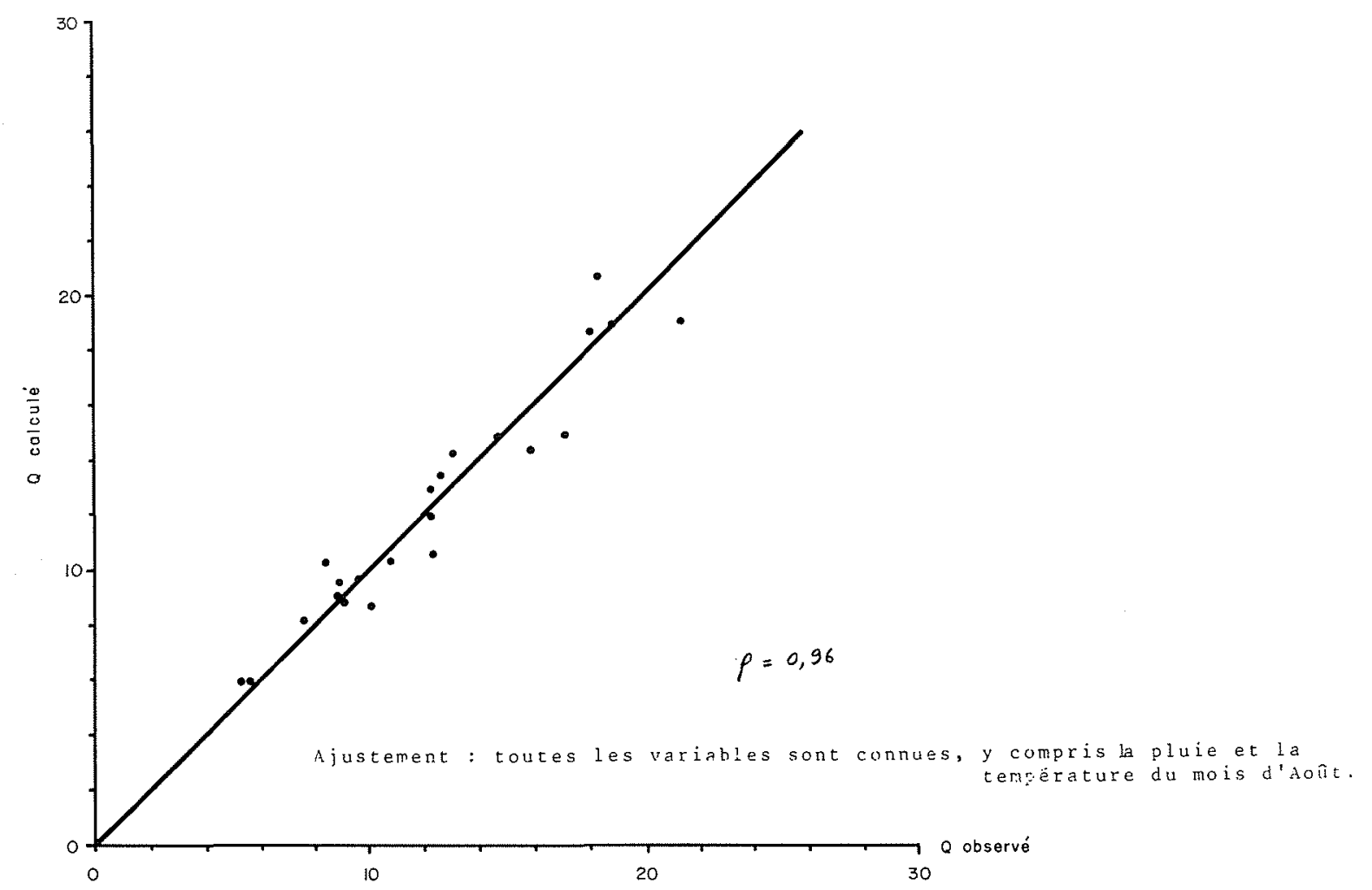

4/ L'Oise à Sempigny. Calcul du débit moyen minimum de 5 jours consécutifs au mois d'Août. Méthode de régression progressive Step-Wise. 


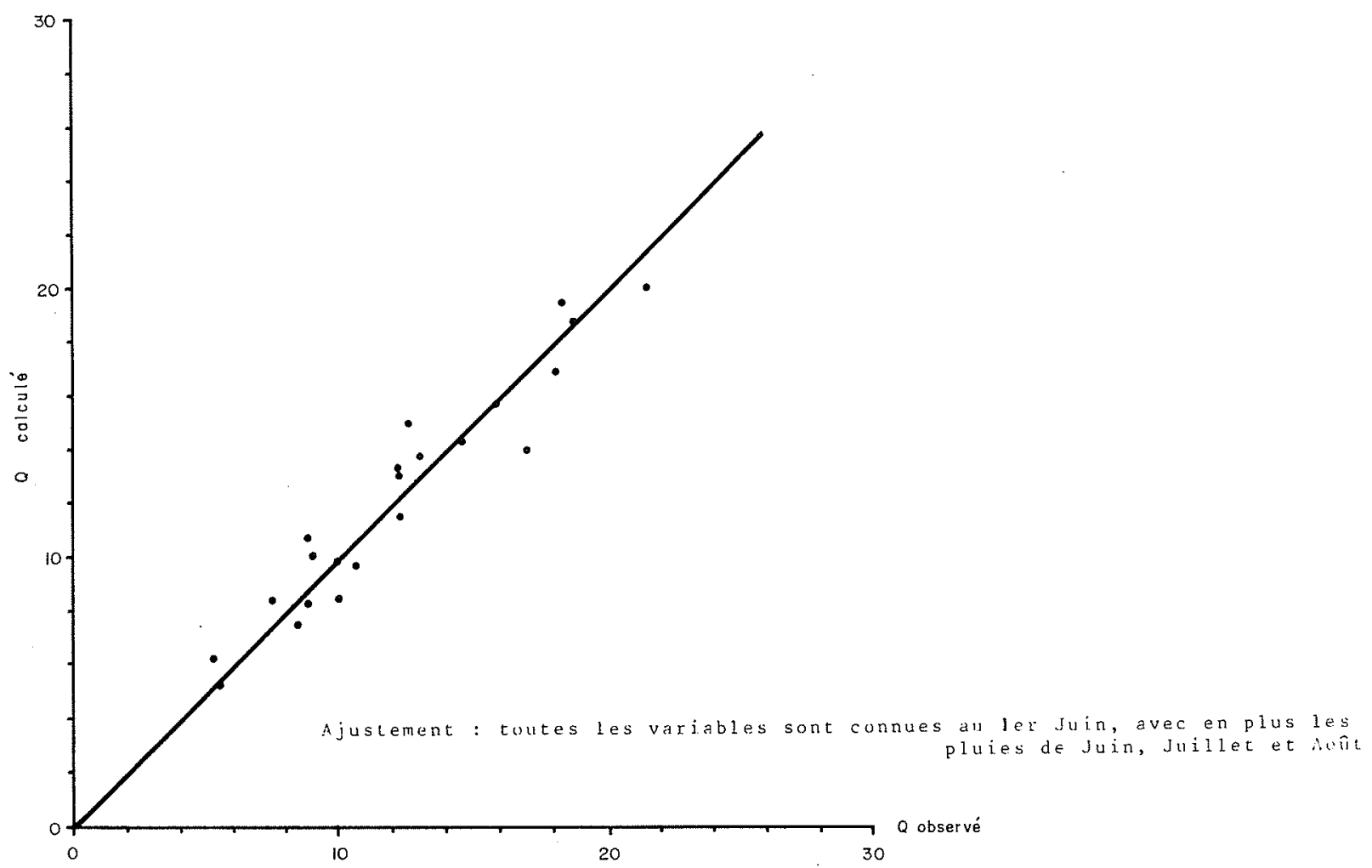

5/ L'Oise à Sempigny. Calcul du débit moyen minimum de 5 jours consécutifs au mois d'Août. Méthode de régression progressive Step-Wise

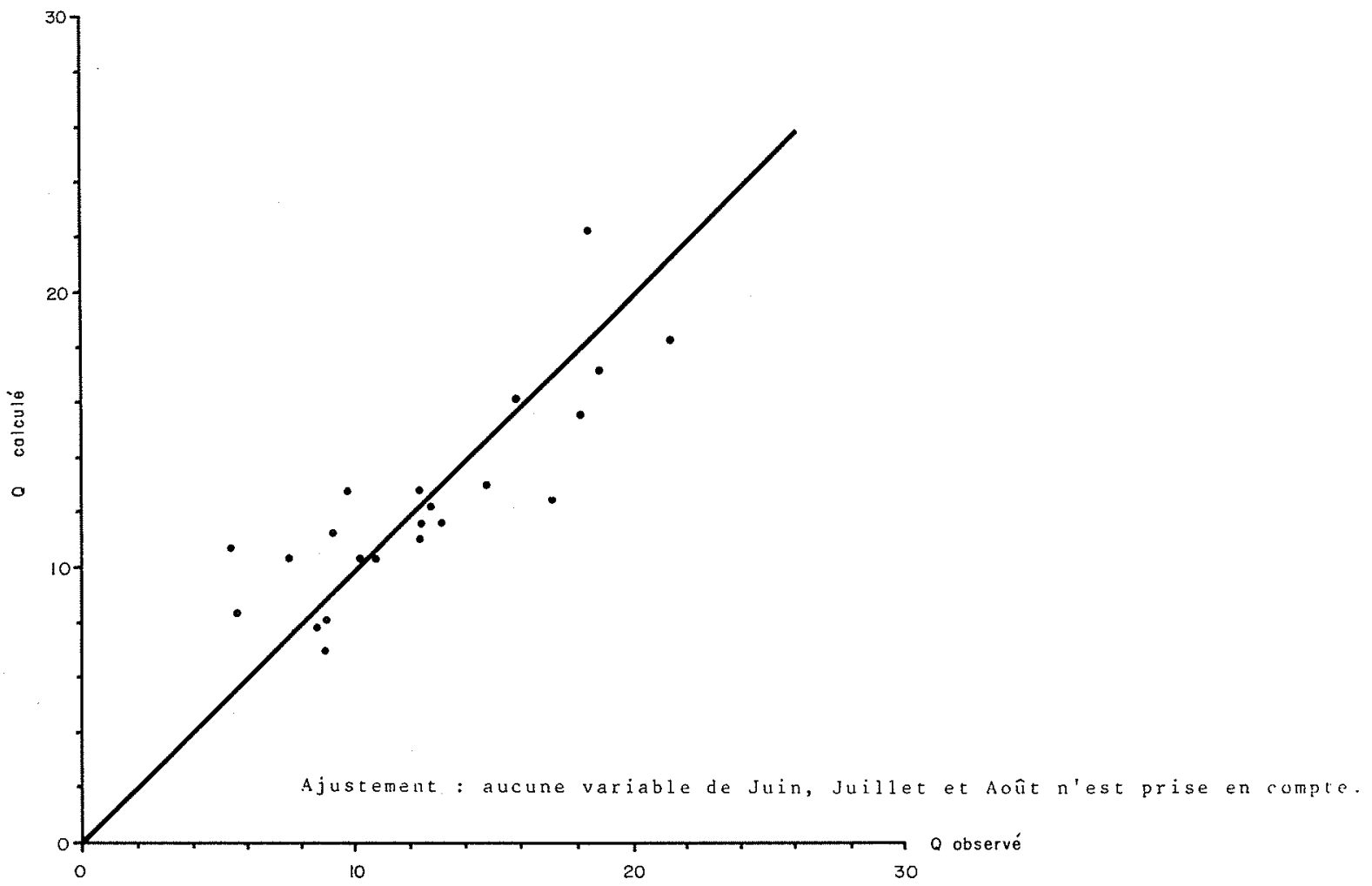

6/ L'Oise à Sempigny. Calcul du débit moyen minimum de 5 jours consécutifs au mois d'Août. Méthode de régression progressive Step-Wise

562 


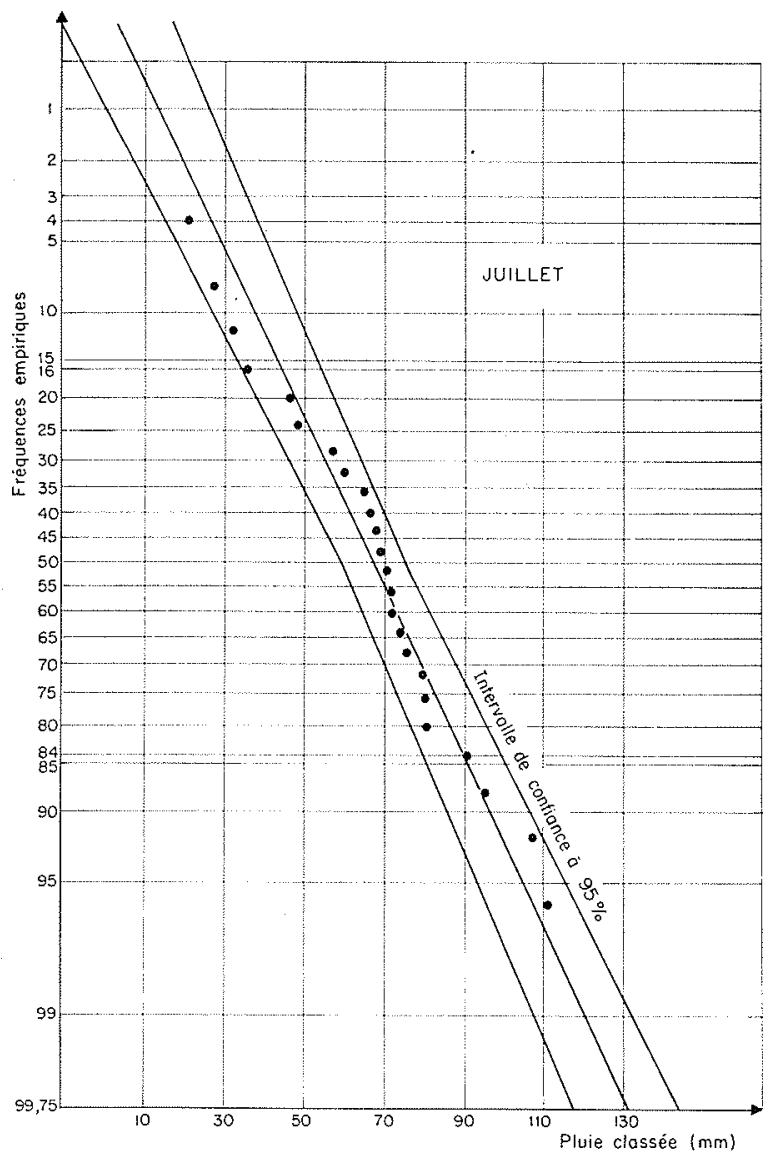

7/ Ajustement d'une loi normale aux précipitations estivales.

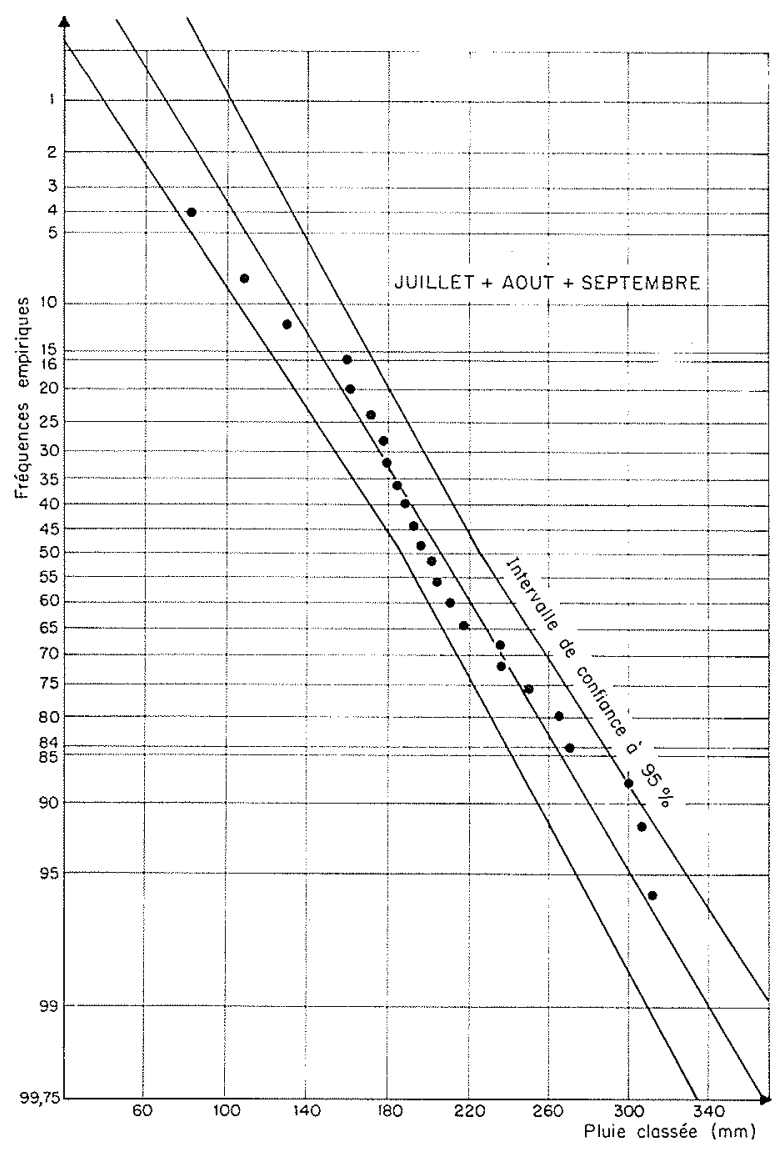

9/ Ajustement d'une loi normale aux précipitations estivales.

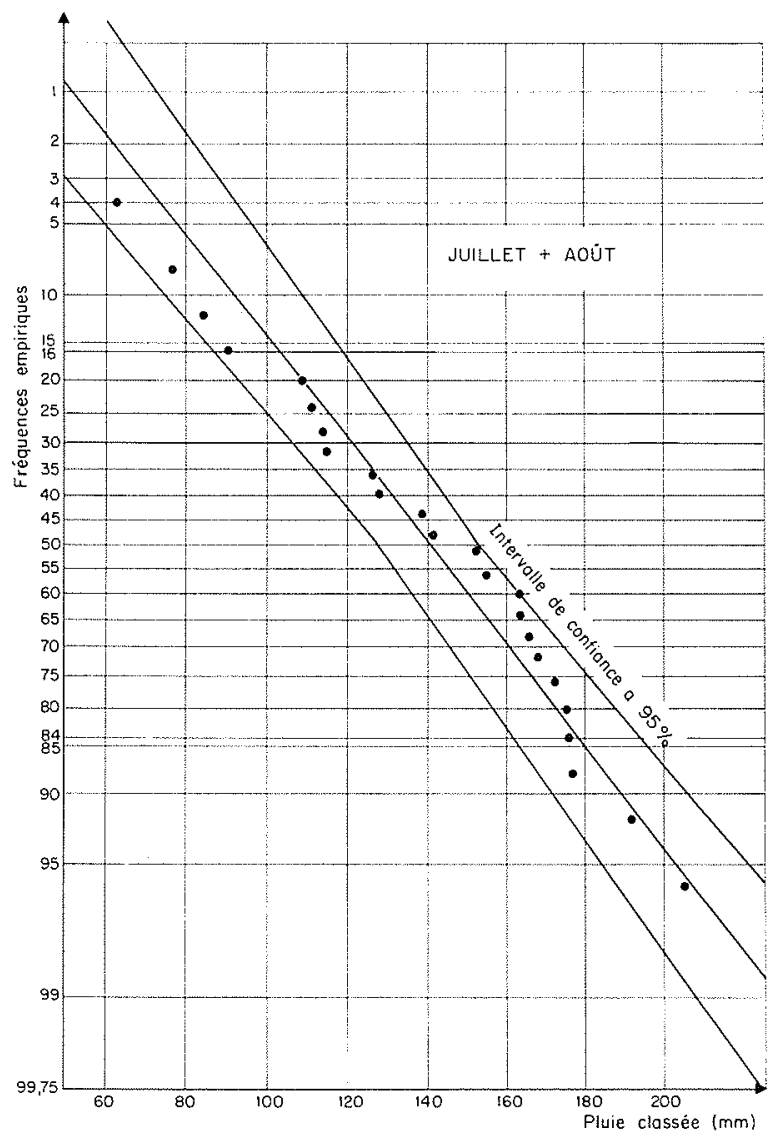

8/ Ajustement d'une loi normale aux précipitations estivales.

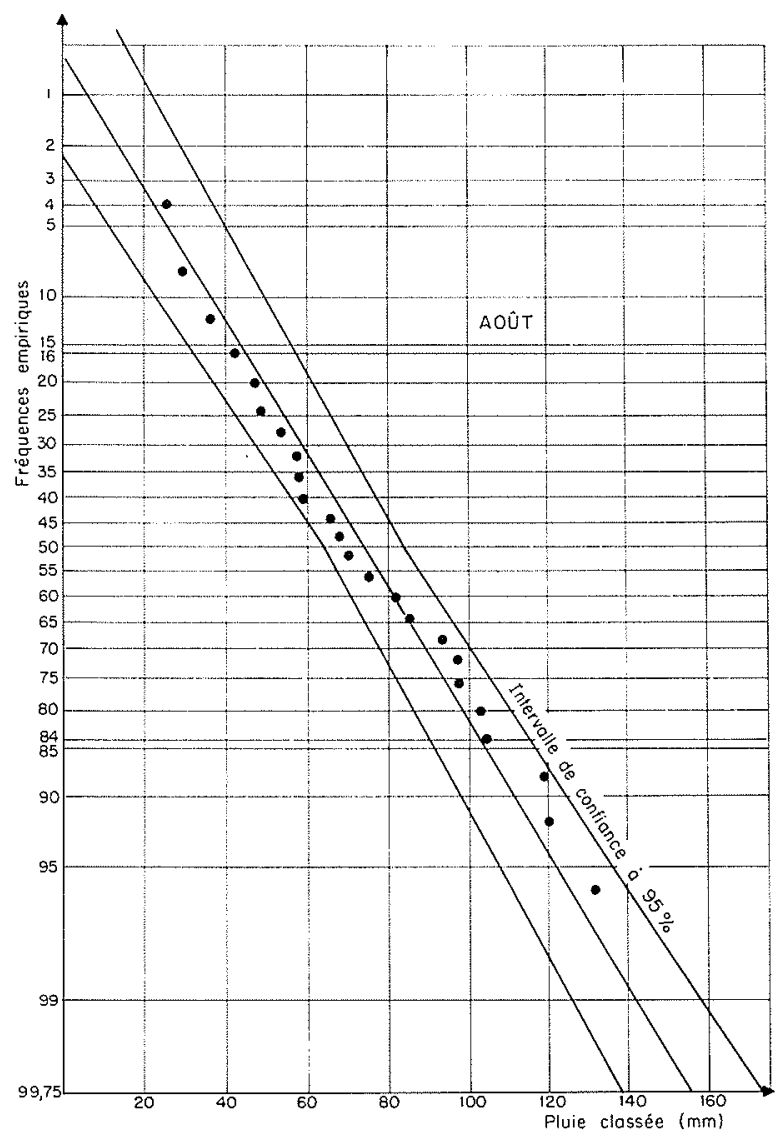

10/ Ajustement d'une loi normale aux précipitations estivales. 


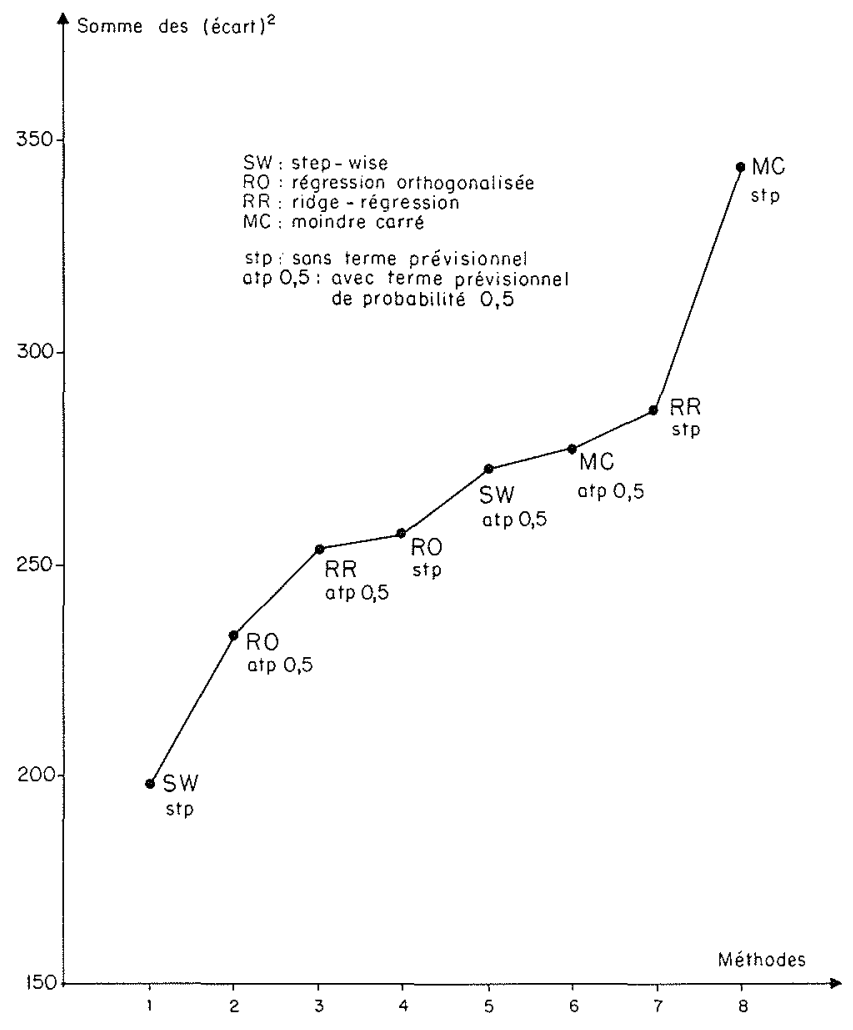

11/ Prévision des $Q_{5}$ du mois d'Août. Période 1931-1949.

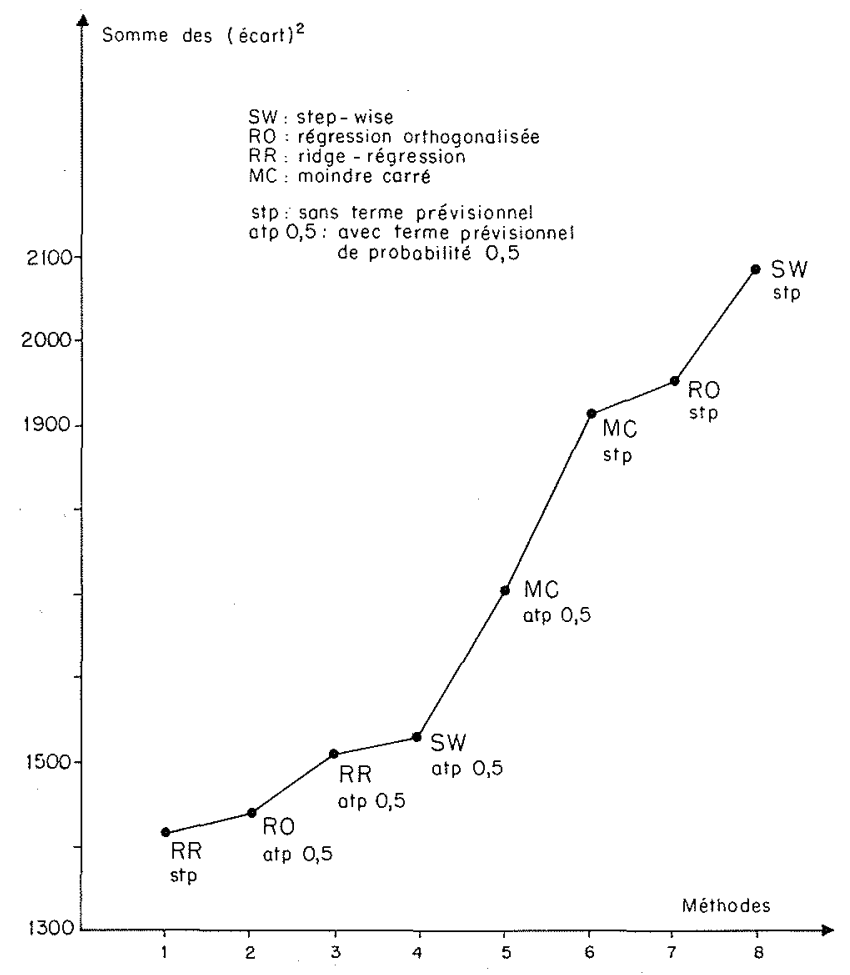

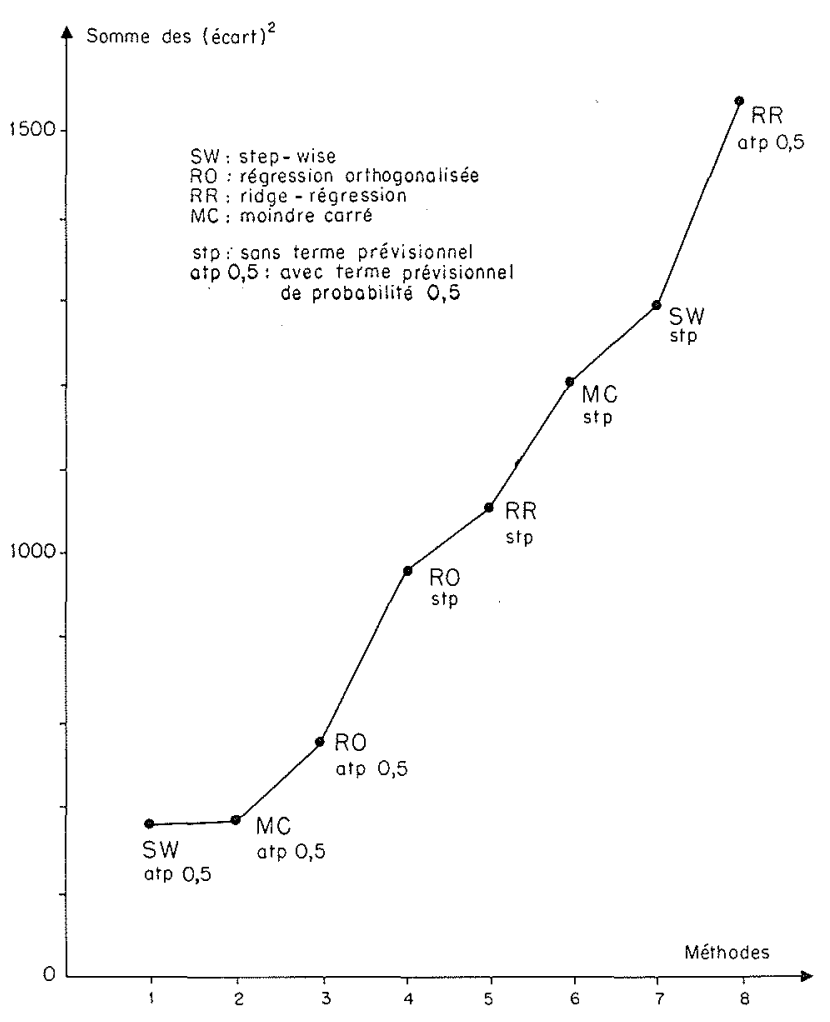

12/ Prévision du débit moyen de Septembre. Périoda 1931. 1949

13/ Prévision du débit moyen d'Octobre. Période 1931-1949. 

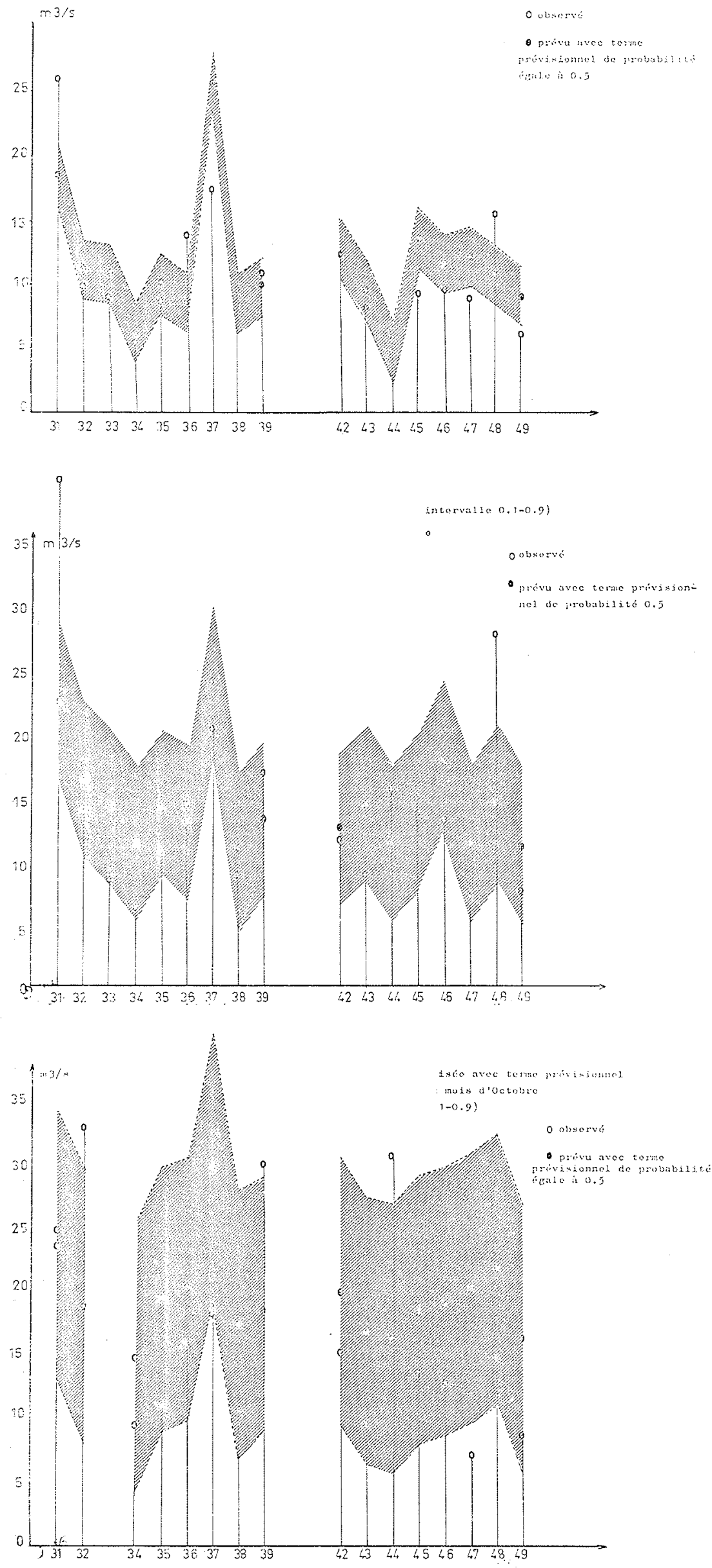

14/ Régression Orthogonalisée avec terme prévisionnel. $O_{5}$ du mois d'Aout (intervalle $0.1-0.9$ ).

15/ Step-Wise avec terme prévisionnel (intervalle $0.1-0.9$ ). Débit moyen du mois de Septembre

16/ Régression orthogonalisée avec terme prévisionnel. Débit moyen du mois d'Octobre (intervalle 0.1-0.9). 


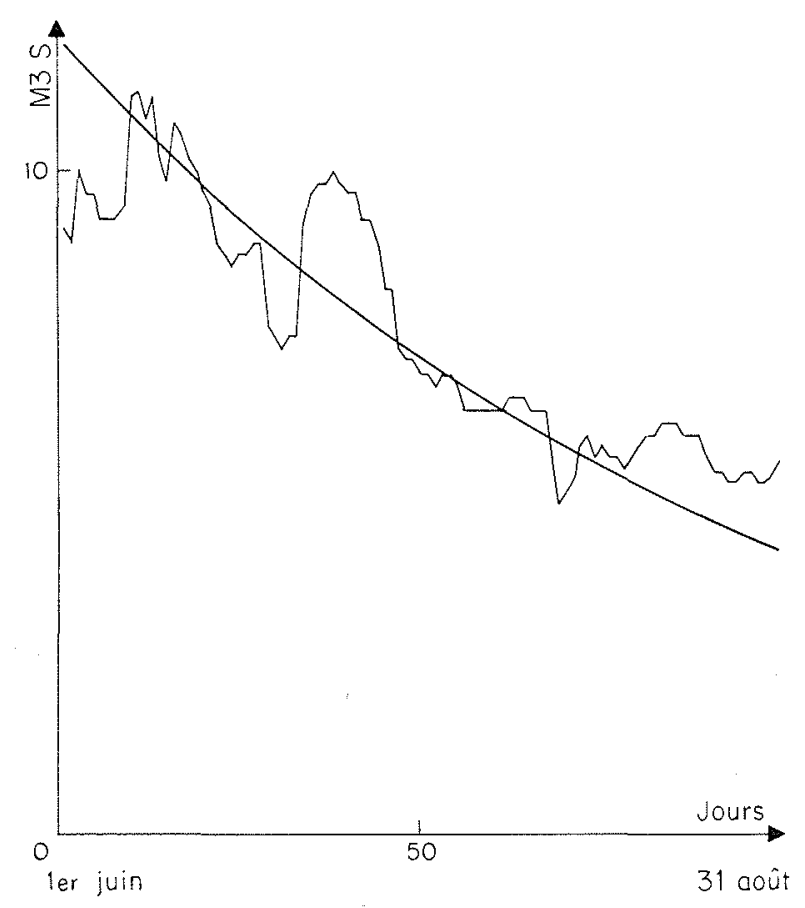

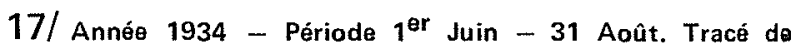
l'exponentielle moyenne (ajustée 1930 -1949).

\begin{tabular}{|c|c|c|c|c|c|c|c|c|c|c|c|c|c|}
\hline \multicolumn{12}{|c|}{ Températures moyennes (moyenne des $\frac{T n+T x}{2}$ quotidiennes) } & \multicolumn{2}{|c|}{ Tableau 1} \\
\hline \multirow{3}{*}{\multicolumn{7}{|c|}{ Station : SAINT-QUENTIN (Fontaine aux Clercs) }} & \multirow{3}{*}{\multicolumn{5}{|c|}{$\begin{array}{ll}\text { Altitude : } & 98 \mathrm{~m} \\
\text { Latitude : } & 40^{\circ} 49^{\prime} \mathrm{N} \\
\text { Longitude : } & 03^{\circ} 12^{\prime} \mathrm{E}\end{array}$}} & & \\
\hline & & & & & & & & & & & & & \\
\hline & & & & & & & & & & & & & \\
\hline Année & Janv. & Fév. & Mars & Avril & Mai & Juin & Juil. & Août & Sept. & Oct. & Nov. & Déc. & Année \\
\hline 1931 & $(3,3)$ & $(3,3)$ & $(4,4)$ & $(7,7)$ & $(12,6$ & $(15,7)$ & $(16,2)$ & $(16,0)$ & $(11,5)$ & $(9,0)$ & $(7,2)$ & $(3,4)$ & $(9,2)$ \\
\hline 1932 & $(4,3)$ & $(0,8)$ & $(4,6)$ & $(7,5)$ & $(11,3)$ & $(14,7)$ & $(16,5)$ & $(19,6)$ & $(15,7)$ & $(9,7)$ & $(6,1)$ & $(4,0)$ & $(9,6)$ \\
\hline 1933 & $(-0,1)$ & $(3,9)$ & 7,5 & 0,0 & 12,5 & 14,3 & 18,1 & 18,5 & 10,7 & 10,7 & 4,4 & 2,6 & 9,4 \\
\hline 1934 & 2,5 & 2,5 & 5,0 & 10,5 & 12,4 & 16,4 & 18,8 & 17,0 & 16,7 & 11,4 & 5,6 & 7,1 & 10,5 \\
\hline 1935 & 2,2 & 4,4 & 5,6 & 8,6 & 11,0 & 16,9 & 18,8 & 17,7 & 15,0 & 10,0 & 6,6 & 2,7 & 9,9 \\
\hline 1936 & 5,0 & 2,8 & 7,3 & 6,8 & 13,0 & 16,5 & 16,4 & 17,5 & 15,0 & 8,4 & 5,5 & 3,0 & 9,8 \\
\hline 1937 & 4,5 & 5.9 & 4,4 & 9,6 & 14,2 & 15,4 & 17,0 & 18,1 & 14,4 & 11,3 & 4,8 & 2,1 & 10,1 \\
\hline 1938 & 4,5 & 3,2 & 8,7 & 6,7 & 10,8 & 15,5 & 17,6 & 17,6 & 15,2 & 9,0 & 9,0 & 1,0 & 9,9 \\
\hline 1939 & 5,1 & 4,2 & 5,1 & 10,0 & 11,6 & 16,0 & 16,2 & 17,4 & 14,0 & 8,9 & 8,3 & 1,7 & 9,9 \\
\hline 1940 & $-4,4$ & 1,6 & 6,6 & 8,9 & $(13,5)$ & $(16,5)$ & $(16,0)$ & $(16,1)$ & $(14,5)$ & $(9,0)$ & $7,0)$ & $(0,4)$ & $8,8^{*}$ \\
\hline 1941 & $(-1,5)$ & $(3,0)$ & $(6,5)$ & $(7,3)$ & $(9,7)$ & $(16,5)$ & $(18,0)$ & $(15,5)$ & $(14,7)$ & $(10,3)$ & $(5,3)$ & $(3,2)$ & $(9,0)$ \\
\hline 1942 & $(-2,7)$ & $(-3,0)$ & $(6,5)$ & $(10,5)$ & $(12,5)$ & $(15,3)$ & $(16,7)$ & $(18,0)$ & $(15,3)$ & $(12,0)$ & $(5,0)$ & $(4,0)$ & $(9,2)$ \\
\hline 1943 & $(4,0)$ & $(4,8)$ & $(7,3)$ & $(10,7)$ & $(13,5)$ & $(15,0)$ & $(18,5)$ & $(17,8)$ & $(14,5)$ & $(11,3)$ & $(5,0)$ & $(2,0)$ & $(10,4)$ \\
\hline 1944 & $(4,5)$ & $(1,3)$ & $(4,7)$ & $(9,8)$ & $(12,0)$ & $(14,3)$ & $(17,3)$ & $(20,0)$ & $(13,5)$ & $(9,3)$ & $(6,3)$ & 3,0 & $(9,7)$ \\
\hline 1945 & $-3,8$ & 6,6 & 8,0 & 10,9 & 14,3 & 16,1 & 18,1 & 17,0 & 15,4 & 12,1 & 5,5 & 3,7 & 10,3 \\
\hline 1946 & $-0,7$ & 5,5 & 5,6 & 10,4 & 12,6 & 13,8 & 17,7 & 15,9 & 15,1 & 10,1 & 6,5 & 0,0 & 9,4 \\
\hline 1947 & $-0,7$ & $-1,9$ & 5,7 & 11,1 & 15,2 & 17,5 & 19,4 & 20,8 & 17,5 & 11,1 & 7,0 & 3,9 & 10,6 \\
\hline 1948 & 5,3 & 3,2 & 8,9 & 10,4 & 13,7 & 15,6 & 16,7 & 16,6 & 14,1 & 9,9 & 6,1 & 3,3 & 10,3 \\
\hline 1949 & 4,2 & 4,9 & 4,9 & 11,5 & 11,6 & 14,7 & 18,8 & 17,8 & 18,5 & 12,3 & 5,3 & 4,0 & 10,7 \\
\hline 1950 & 1,7 & 6,4 & 6,9 & 8,2 & 13,8 & 17,6 & 18,2 & 17,4 & 13,9 & 9,9 & 6,4 & $-0,9$ & 10,0 \\
\hline 1951 & 4,5 & 4,4 & 5,3 & 8,2 & 11,9 & 15,3 & 17,2 & 16,6 & 15,0 & 9,4 & 8,3 & 3,7 & 10,1 \\
\hline 1952 & 2,2 & 2,1 & 7,3 & 11,3 & 14,0 & 16,1 & 18,4 & 17,9 & 11,7 & 9,4 & 3,7 & 2,3 & 9,7 \\
\hline 1953 & $-0,4$ & 2,8 & 7,1 & 9,8 & 14,2 & 15,2 & 17,0 & 17,8 & 15,2 & 11,7 & 5,7 & 5,9 & 10,2 \\
\hline 1954 & 0,2 & 0,9 & 7,5 & 7,7 & 12,8 & 14,9 & 14,9 & 16,2 & 14,2 & 12,4 & 6,8 & 5,8 & 9,5 \\
\hline 1955 & 2,3 & 1,6 & 3,4 & 9,4 & 11,3 & 15,6 & 18,2 & 18,1 & 14,5 & 9,9 & 6,5 & 5,0 & 9,7 \\
\hline 1956 & 3,5 & $-6,4$ & 6,7 & 7,3 & 13,3 & 13,0 & 16,7 & 14,9 & 16,1 & 10,2 & 4,7 & 5,6 & 8,8 \\
\hline 1957 & 2,8 & 6,6 & 9,4 & 8,9 & 10,7 & 16,6 & 18,1 & 16,3 & 13,8 & 10,9 & 6,0 & 2,9 & 10,3 \\
\hline 1958 & 3,1 & 5,0 & 3,6 & 7,0 & 13,0 & 14,9 & 16,9 & 17,3 & 16,3 & 11,1 & 5,4 & 4,7 & 9,0 \\
\hline 1959 & 2,1 & 2,5 & 8,0 & 10,0 & 13,7 & 16,2 & 19,2 & 18,5 & 16,8 & 11,7 & 5,8 & 5,2 & 10,8 \\
\hline 1960 & 2,9 & 4,1 & 7,4 & 9,1 & 14,2 & 16,3 & 15,7 & 16,5 & 13,9 & 10,8 & 8,0 & 2,9 & 10,1 \\
\hline
\end{tabular}




\section{Discussion}

President : M. M. CAR LIE R

M. le Président remercie M. Masson pour son exposé qui traite d'un problème original et difficile en utilisant des méthodes statistiques avancées. Il ouvre ensuite la discussion.

M. TIXERONT fait la suggestion suivante :

M. MASSON signale qu'il y a peu de relation entre la température d'avril et le débit d'étiage de l'été suivant. Ne serait-il pas plus intéressant de se baser plutôt que sur la température, sur l'excédent ou le déficit de la pluie par rapport à l'évaporation potentielle ? On ferait ainsi intervenir la combinaison : "pluie-températurecouverture végétale.

M. MASSON remercie M. TIXERONT de sa proposition qu'il compte bien "tester".

Quel est l'ordre de grandeur :

1) de l'erreur de prévision (définie de façon statistique) ?

2) des coefficients de corrélations multiples? demande M. BERNIER.

Ces ordres de grandeur sont très différents sujvant les variables et les mois considérés, répond M. MASSON. A titre d'exemple, je signale, de mémoire, que pour le débit moyen minimum de 5 jours consécutifs du mois d'Août, prévu uniquement à partir du débit moyen de 5 jours consécutifs du mois de mai, on a un coefficient de corrélation de 0,83 .

M. DUBREUIL pose les questions ci-après :

Le gradient de variation de la pluviosité avec l'altitude est-il indépendant de l'orientation par rapport aux vents dominants. Ce gradient varie-t-il d'un mois à l'autre et existe-t-il également durant les mois d'été où les pluies d'orage sont majoritaires ?

A propos des gradients de pluviosité, M. BEDrot indique que, dans la partie du bassin de l'Oisc orientée vers le Sud-Ouest, on a appliqué un gradient moyen déduit d'études faites sur divers bassins de Seine-Normandie.

Dans le Morvan, on distingue trois zones de gradients : une zone orientée à l'ouest avec un très fort gradient ; une zone orientée vers l'est (sous le vent) qui donne des gradients moins importants ; enfin, la couronne nord qui présente des gradients intermédiaires.

L'utilisation des moyens informatiques pour l'exploitation de fichiers, de plus en plus complets, permet de calculer avec une bonne approximation la "lame d'eau précipitée" pour telle ou telle partic du bassin ; dans ces conditions, l'emploi de la méthode de Thiessen est moins avantageux que lorsque l'on ne disposait que de quelques stations pour l'ensemble du bassin. On pourra parfois estimer cette lame par la moyenne arithmétique des observations.
M. Gull LOT s'interroge sur l'influence positive - d'après l'équation de regression - de la température des mois de printemps (mars avril surtout) sur l'étiage du mois d'août.

Il serait d'abord prudent de vérifier s'il n'y a pas, par hasard, sur l'échantillon d'années une corrélation positive fortuite entre la température de Mars-Avril et les précipitations de Juillet-Août.

Bien que l'on puisse être, a priori, inquiet, dit-il, sur la signification des coefficients de régression ajustés sur 20 ans seulement d'observations avec un nombre élevé de variables explicatives :

l'influence positive qui a été trouvée de la tempéature des mois de printemps, ne peut-elle s'expliquer, par exemple, par une évolution plus rapide de la végétation d'où une consommation moindre en eau des végétaux dans la deuxième partie de l'été ? Qu'en pensent les agronomes?

Les pluies d'été ayant peu d'effet sur l'écoulement, observe M. de BEAUREGARD, il parait difficile d'estimer, dès le $1^{\text {er }}$ août, l'écoulement d'octobre. Quel degré de liaison relève-t-on entre ces pluies d'été et ces débits d'octobre?

Le débit moyen d'octobre, répond M. Masson, dépend pour l'essentiel des pluies qui tomberont entre le $1^{\text {er }}$ aoút et le mois d'octobre; l'intervalle de confiance afférent à cette prévision reflète l'incertitude qui y est attachée. Classiquement, le bilan hydrique d'un bassin versant est dans son ensemble négatif durant les mois d'oût et de septembre, remarque M. TIXERONT'; mais en certains points : zones urbanisées, routes revêtues, il est en réalité positif, et après les averses, il y a dans ces zones un certain ruissellement. Cela explique que les débits commencent à augmenter au début d'octobre et même en septembre.

M. DUBAND souligne, en ces termes, quelques précautions à prendre dans ce genre d'étude :

Ainsi que l'a indiqué M. MAsson, l'utilisation des techniques de corrélation multiple ne peut s'effectuer par des programmes appliqués automatiquement ; elle nécessite un contrôle sérieux des données historiques, une analyse détaillée des résultats d'essais de calculs de régressions multiples, éventuellement l'utilisation de techniques concurrentes (ridge regressions, etc.) pour éprouver la stabilité et la robustesse des équations de prévision. On doit chercher également à appuyer ces résultats statistiques sur des considérations physiques ; dans le cas particulier de l'Oise, les résultats obtenus pour la prévision du débit minimum d'août sont cohérents avec l'application de la méthode semi-statistique de la "charnière", probablement du fait de la faiblesse des précipitations de Juillet et Août. 


\section{Abstract \\ Experimental forecasts of low-water conditions in the river Oise at Sempigny}

As a result of the present heavy demand for water and increasing pollution of water supplies, forecasting of low-water conditions has become a vital necessity. At the request of the "Agence Finan cière de Bassin Seine-Normandie", a number of forecasting methods were tried out by the "SMERS" establishment. The test location was at Sempigny on the river Oise, which has a catchment area of $4290 \mathrm{~km}^{2}$. Particulars of the latter, which is unaffected by storage ponds or reservoirs, are shown in Fig. 1 and Table 1.

The experimental forecasts related to the following :

a) Minimum mean discharge for all series of five and ten consecutive days in a month

b) Minimum mean monthly discharge.

Conditions were forecast two months ahead.

Available data

Daily discharge data for Sempigny (since 1955).

Daily stage data at Sempigny (since 1892), which were converted to daily discharge data.

Daily rainfall data from an average number of fifteen gauging stations (see Fig. 5), from which monthly rainfall in the catchment area and the probability of summer rainfall were calculated.

Mean monthly temperature at Saint Quentin.

Forecasting aims and methods

The forecasts were to enable prediction of the following flow characteristics in a given month $J$ two months ahead, i.e. during the first week of month $J-2$ :

$Q^{5} \quad$ : minimum mean discharge over five consecutive days

$Q^{10}$ : minimum mean discharge over ten consecutive days

$Q^{m}$ : mean monthly discharge.

In other words, $Q^{5}, Q^{10}$ and $Q^{m}$ for August were calculated during the first week of June, those for September in the first week of July, and so on, using the following methods :

a) Entirely stochastic methods

b) Partly deterministic methods

For the stochastic approach a least-squares method was tried, which gives minimum residual variance for the sample of adjustment.
Ridge regression and orthogonalized regression methods were also attempted, which gave better forecast data than the least-squares method. "Forecasting terms", which are unknown at the time of making the forecast and minimize residual variance, were applied. Stopwise and cross-validation approaches were also tried out.

For the partly deterministic approaches, daily mean discharges over three consecutive months were expressed in terms of an exponential law which is an alternative form of Roche's theory, giving a single-point forecast. This was converted into a forecasting interval of a definite probability. Use was also made of Bernier's characteristic flow theory and a deterministic model at the Electricite de France Research and Test Establishment (CREC).

Results

Adjustments :

Table 2 shows the steps in which adjustment was effected. Column a : all variates are known

Column $b$ : precipitation is assumed to be known throughout the considered period. Discharge and temperature in months $J .2$ and $J-1$ are not known.

Column $\mathrm{c}$ : no forecasting terms

Examples are shown in Tables 3 and 4 , and the exponential on graph 1.

Forecasts

The methods were tried out on a series which was not considered for adjustment purposes. The results are listed in Tables 5 et seq.

\section{Conclusion}

These first results are considered to be quite promising, since they show that it is feasible to give a guaranteed forecast of minimum discharge two months ahead. Regular forecasting by the methods described is scheduled to start in June 1976. 